J. Urol. Urogynäkol. AT 2021 · 28:58-72 https://doi.org/10.1007/s41972-021-00134-w Angenommen: 17. März 2021 Online publiziert: 19. April 2021

๑) Der/die Autor(en) 2021
Michael Grunert ${ }^{1,2} \cdot$ Nina Eberhardt ${ }^{1} \cdot$ Vikas Prasad $^{1} \cdot$ Ambros J. Beer $^{1}$

' Klinik für Nuklearmedizin, Universitätsklinikum Ulm, Ulm, Deutschland

${ }^{2}$ Klinik für Nuklearmedizin, Bundeswehrkrankenhaus UIm, Ulm, Deutschland

\title{
Nuklearmedizinische Diagnostik und Therapie des Prostatakarzinoms
}

\section{Wer braucht wann ein PSMA-PET? Gibt es einen Stellenwert für die PSMA-Therapie?}

\section{Hintergrund}

Das Prostatakarzinom ist eine der am häufigsten diagnostizierten Krebsarten weltweit, mit einer geschätzten globalen Inzidenz von etwa 1.276.100 Fällen im Jahr 2018. Die geschätzte Zahl der Neuerkrankungen in den USA liegt bei ca. 174.650 und ist mit ca. 31.620 Todesfällen pro Jahr die zweithäufigste Krebstodesursache in den Vereinigten Staaten. In Deutschland lag die Prognose für das Jahr 2020 bei 61.200 Neuerkrankungen und etwa 14.320 Todesfällen. In Österreich betrug die Inzidenz 2017 ca. 5700 Neuerkrankungen, und 1260 Männer starben daran [1-4].

Die nuklearmedizinische Diagnostik ist für die Festlegung der am besten geeigneten Therapie im Primärstaging und insbesondere im Rezidiv von besonderer Bedeutung. Für die Stratifizierung ist eine exakte prä-, interund posttherapeutische Bildgebung essenziell (• Abb. 1). Im letzten Jahrzehnt nahm die Positronen-EmissionsTomographie/Computertomographie (PET/CT) eine immer größere Rolle als bildgebendes Hybridverfahren ein und gewinnt mit der klinischen Einführung des prostataspezifischen PSMALiganden 68Ga-PSMA-11 im Jahr 2011 immer mehr an Bedeutung für die individualisierte Behandlung. Die Anzahl

Die Autoren M. Grunert und N. Eberhardt haben zu gleichen Teilen zum Manuskript beigetragen. der Publikationen zeigen den Anstieg der Bedeutung des PSMA-PET in der Diagnostik (• Abb. 2).

Ga68-PSMA übertrifft die Detektionsraten der zuvor lange genutzten C-11Acetat und C-11-Cholin PET-Tracer, die aufgrund der unspezifischen Aufnahme in gutartigen Läsionen vorrangig in der Rezidivdiagnostik eingesetzt wurden [5]. Das bei der überwiegenden Anzahl bei onkologischen Fragestellungen genutzte 18F-FDG hat bei der Diagnostik des Prostatakarzinoms lediglich einen untergeordneten Stellenwert und kommt nur bei aggressiven sowie schlecht differenzierten Karzinomen oder therapieassoziierter neuroendokriner Differenzierung mit erhöhter Glukosestoffwechselaktivität in Betracht.

\section{PSMA-Liganden}

Das PSMA-spezifische Membranantigen ist aktuell das erfolgreichste Target in der Bildgebung und in der nuklearmedizinischen Therapie. Es ist ein membrangebundenes Glykoprotein, auch Glutamatcarboxypeptidase II genannt, das von Prostatakarzinomen und deren Metastasen deutlich überexprimiert wird [6]. Durch die Überexpression in 90-100 \% der Prostatakarzinomzellen ist PSMA daher eine optimale Zielstruktur mit Zunahme der Hochregulierung bei höherem Gleason-Score, als Ausdruck der Entdifferenzierung und unter antiandrogener Therapie (ADT; [7]). Der
PSMA-Ligand 68Ga-PSMA-11 zählt weltweit zu den am häufigsten genutzten klinischen Tracern [8].

Anders als der Name vermuten lässt, ist eine PSMA-Expression auch abseits der Prostata und des Prostatakarzinoms in anderen Organen physiologisch [9-12] und wird auch in benignen und malignen Tumorentitäten gefunden [13]. In einer Vielzahl von Malignomen, wie den Gliomen, Kopf-Hals-Malignomen, Schilddrüsen-, Lungen- und Brustkarzinomen sowie im hepatozellulären Karzinom, Nierenzellkarzinom, Lymphom und Melanom wird eine erhöhte PSMA-Liganden-Anreicherung beobachtet. Die physiologischen Speicherungen der PSMA-Liganden sind in - Tab. 1 und - Abb. 3 aufgezeigt.

Die seit 2011 stetige Weiterentwicklung der PSMA-Liganden führte basierend auf der klinischen Nachfrage nach zielgerichteter Bildgebung zu einer Vielzahl an Ga68- und F18- markierten PSMA-Liganden. 68Ga-PSMA wird vermehrt durch F18-markierte Liganden ersetzt. Die Einschränkungen von 68Ga-PMSA mit einer Halbwertszeit von 68 min und der Verfügbarkeit von nur 2-4 Patientendosen pro Herstellungsvorgang wird durch F18-PSMA mit einer Halbwertszeit von $110 \mathrm{~min}$ und höheren Produktionskapazität durch die Zyklotronproduktion ausgeglichen [14]. Einige 18F-markierten PSMA-Liganden, wie das F18-PSMA-1007, zeigen eine reduzierte Urin-Clearance und eigenen 


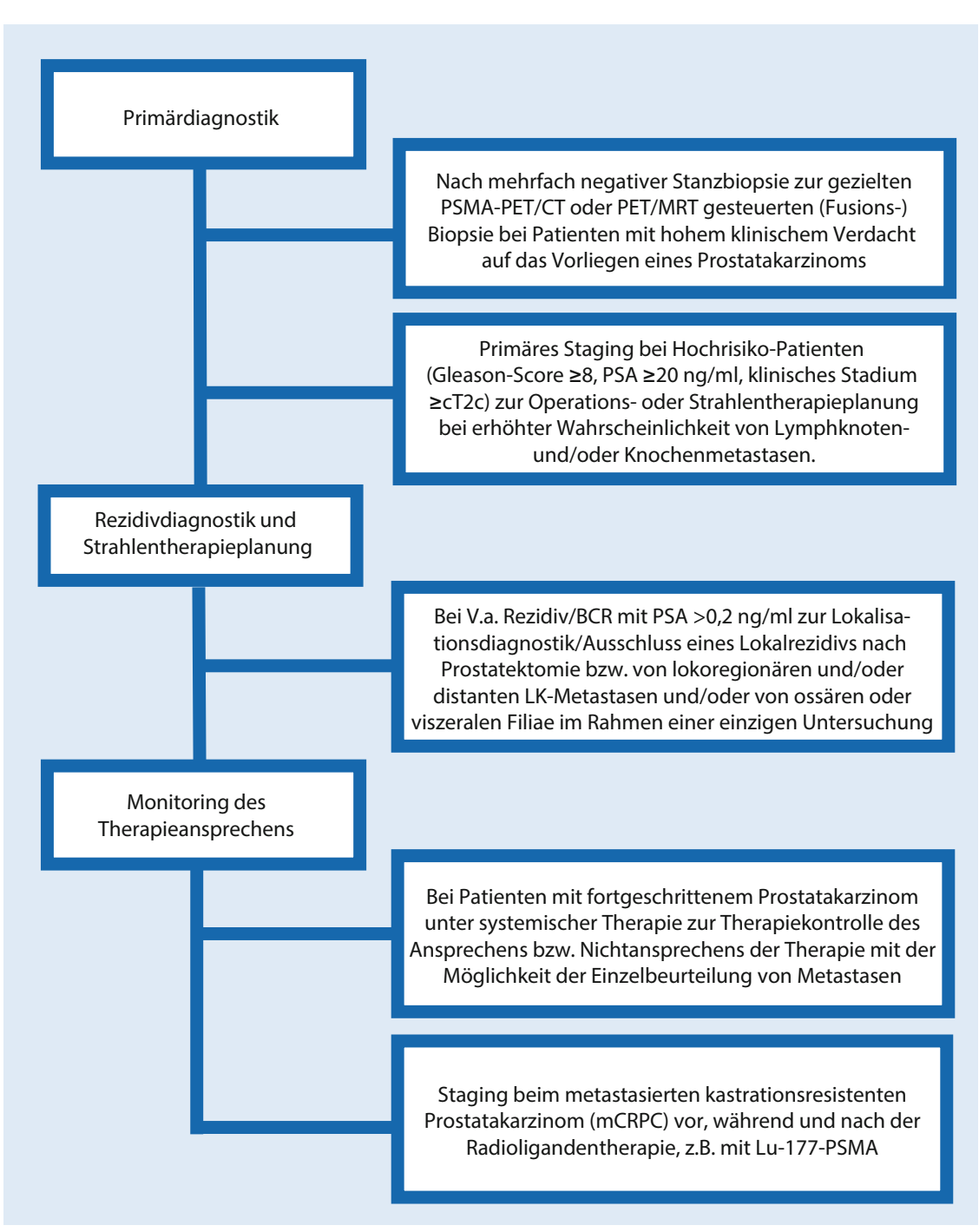

Abb. 1 ॥ Einsatzmöglichkeiten und Indikationen der PSMA-PET in den unterschiedlichen Erkrankungsstadien

sich besonders zur Detektion eines Lokalrezidivs bzw. für die lokoregionäre Tumorausbreitung ([15]; Fallbeispiel in - Abb. 4).

Die Frage, ob 68Ga- oder 18F-PSMA in der Diagnostik verwendet werden sollte, ist aktuell noch nicht abschließend geklärt; sie sollten für die meisten klinischen Indikationen als austauschbar angesehen werden und sich eher an den lokalen Gegebenheiten ausrichten.

\section{Diagnose und Primärstaging}

Für die Planung des weiteren therapeutischen Procederes sind genaue Kenntnisse über die Ausdehnung des Primarius, insbesondere eine extraprostatische Ausbreitung und die Infiltration in Nachbar-
Risiko- und stadienadaptiert wird in der Leitlinie weiterhin die Skelettszintigraphie, CT und Magnetresonanztomographie (MRT) empfohlen. In aktuelleren Studien zeigen sich vielversprechende Ergebnisse für den Einsatz des PSMA-PET im primären Staging.

Die multiparametrische MRT der Prostata (mpMRT) zeichnet sich gegenüber der PET/CT durch einen höheren Weichteilkontrast insbesondere in der hochaufgelösten T2w- und in den kontrastmittelverstärkten Sequenzen (DCE) aus und wird ergänzt durch die Diffusionswichtung als funktionelle Komponente. Die Kombination aus MRT und der PSMA-PET im Rahmen einer simultanen PSMA-PET/MRT bei Intermediate- und High-risk-Prostatakarzinomen zeigte eine höher diagnostische Genauigkeit in der Detektion des Prostatakarzinoms unter Verwendung der PIRADS-Kriterien bzw. einer 5-Punkte-Likert-Skala mit histopathologischer Korrelation. Die mpMRT, PET und PET/MRT detektierten in 66, 92 und $98 \%$ das Prostatakarzinom. Die kombinierte PET/MRT-Untersuchung (AUC 0,88) war statistisch signifikant der alleinigen PET (AUC 0,83) und mpMRT (AUC 0,73) überlegen [17]. Diese Studienergebnisse zeigen einen möglichen Einsatz im Rahmen der zielgerichteten Fusionsbiopsie auf. In einer weiteren retrospektiven Studie mit gesicherten High-risk-Karzinomen konnte die PSMA-PET/CT 89\% der im mpMRT auffälligen Läsionen detektieren, und vice versa wurden $97 \%$ der auffälligen PET/CT-Läsionen in der MRT erkannt [18].

Beim primären Staging des N- und M-Stadiums bei Patienten mit mittlerem und hohem Risiko hat sich schon früh in klinischen Studien gezeigt, dass die Sensitivität und Spezifität der PSMA-PET/CT die konventionellen Bildgebungsmethoden übertreffen. Die Sensitivität, Spezifität und die diagnostische Genauigkeit der Lymphknoten(LK)-Detektion betrug bei der PSMA-PET 66, 99 und 89\% im Vergleich zur konventionellen Bildgebung mit CT und MRT 44, 85 und $72 \%$ [19]. Dies ist hauptsächlich auf Beurteilung der LK in der CT oder MRT zurückzuführen, da als einziges Kriterium die 
Größe und Morphologie des LK eingeht und diese erst ab $8-10 \mathrm{~mm}$ als suspekt gewertet werden. Der Großteil der Metastasen, die sich unter $8 \mathrm{~mm}$ bewegen, werden somit nicht erfasst und führen zu der geringen Sensitivität.

Für die Detektion von Knochenmetastasen gilt dies analog, die Sensitivität und Spezifität beträgt für die PET 98,8-100\% und 98,9-100\% und in der Szintigraphie 82,4-86,6\% sowie 91,6-97,9\% (Range durch optimistische und pessimistische Klassifikation der Läsionen; [20]). Die bisher erhobenen Studienergebnisse konnten in einer erst kürzlich publizierten prospektiven Studie bei High-riskKarzinompatienten eindrücklich untermauert werden. Die PSMA-PET/CT hat eine $27 \%$ höhere diagnostische Genauigkeit im Vergleich zur konventionellen Bildgebung mit einer Sensitivität und Spezifität von 85 und $98 \%$ vs. 38 und $91 \%$. Die Autoren schlussfolgern, dass die PSMA-PET/CT geeignet ist, die konventionelle Bildgebung mittels CT und Knochenszintigraphie zu ersetzen [21].

\section{Monitoring des Therapie- ansprechens und Restaging im biochemischen Rezidiv}

Zwischen 27 und 53\% aller Prostatakarzinompatienten erleiden nach radikaler Prostatektomie (RP) oder Strahlentherapie ein ansteigendes PSA. Beim biochemischen Rezidiv (BCR) ist eine frühzeitige Läsionsdiagnostik mit präziser Aussage zur Tumorausdehnung und Lokalisation essenziell, um die Patienten der besten Therapie zuzuführen, welchelokale Salvage-Strahlentherapie, Salvage LKDissektion oder Einleitung einer Systemtherapie bei polytoper oder disseminierter Metastasierung umfasst [22].

Patienten mit einer biochemischen Krankheitspersistenz 6 Wochen nach primärer Therapie (PSA $>0,2 \mathrm{ng} / \mathrm{ml}$ ) haben ein schlechteres Outcome im Vergleich $\mathrm{zu}$ Patienten, die ein postoperatives PSA unterhalb der Nachweisgrenze aufweisen. Diese Patientengruppe benötigt eine gezielte Diagnostik, um nichttherapiertes Tumorgewebe aufzuspüren und gezielt therapieren zu können. Bei niedrigen PSA-Werten versagte bisher die konventionelle Bildgebung mit CT,

J. Urol. Urogynäkol. AT 2021 · 28:58-72 https://doi.org/10.1007/s41972-021-00134-w (c) Der/die Autor(en) 2021

\section{Grunert · N. Eberhardt · V. Prasad · A. J. Beer \\ Nuklearmedizinische Diagnostik und Therapie des Prostatakarzinoms. Wer braucht wann ein PSMA-PET? Gibt es einen Stellenwert für die PSMA-Therapie?}

\section{Zusammenfassung}

Die PSMA-PET/CT hat durch die präzise Darstellung der Tumorausdehnung einen festen Stellenwert in der Diagnostik, insbesondere in der Rezidivsituation, eingenommen und ist bereits in mehreren nationalen und internationalen Leitlinien fest verankert. Sie ermöglicht, in einem Untersuchungsgang Informationen über die Tumorsituation in der Prostataloge und von potenziellen lymphonodalen, viszeralen und ossären Metastasen zu erlangen, die für die zunehmend personalisierten Behandlungsstrategien notwendig sind. Die PSMA-Therapie stellt bereits jetzt - trotz bisher fehlender Zulassung - eine ergänzende nebenwirkungsarme Therapie beim metastasierten kastrationsresistenten Prostatakarzinom dar, die die Lebensqualität der Patienten deutlich verbessern und die Überlebenszeit steigern kann ohne relevante Toxizität, und deren Potenzial für die Zukunft auch durch Kombination mit anderen Therapieverfahren noch lange nicht absehbar ist. Der Beitrag thematisiert die Einsatzgebiete der PSMA-PET-Bildgebung als Grundlage für die erfolgreiche Therapie sowie den aktuellen Stand zur Indikation, Durchführung und Entwicklung der PSMATherapie.

\section{Schlüsselwörter}

Prostata-spezifisches Membranantigen (PSMA) · Positronen-Emissions-Tomographie/Computertomographie (PET/CT) . Theranostik · Biochemisches Rezidiv · Radioligandentherapie

\section{Nuclear medical diagnostics and treatment of prostate cancer. Who needs PSMA-PET imaging and when? Is there a need for PSMA therapy?}

\section{Abstract}

The PSMA-PET/CT imaging has become firmly established in the diagnostics, especially in recurrency situations, due to the precise imaging of the tumor spread and is already firmly anchored in several national and international guidelines. In a single examination it enables information to be obtained on the tumor situation in the prostate bed and on potential lymph node, visceral and osseous metastases, which are necessary for increasingly personalized treatment strategies. The PSMA treatment already represents - despite the lack of approval to date-an additional treatment with few side effects in metastatic castration-resistant prostate cancer, which can significantly improve the patients' quality of life and increase survival time without relevant toxicity. Its potential for the future also in combination with other treatment methods is not by any means foreseeable. This article addresses the applications of PSMA-PET imaging as a basis for successful treatment as well as the current status regarding indications, implementation and development of PSMA treatment.

\section{Keywords}

Prostate-specific membrane antigen (PSMA) . Positron-emission tomography/computed tomography (PET/CT) - Theranostics . Biochemical recurrence $\cdot$ Radioligand therapy
MRT und Knochenszintigraphie oft. Die Stärke der PSMA-PET wurde in einer multizentrischen retrospektiven Studie herausgehoben. Bei 191 Patienten mit einem medianen PSA von $1,1 \mathrm{ng} / \mathrm{ml}$ wurde in $68 \%$ der Patienten die Metastase(n) lokalisiert, 35\% der Metastasen waren im Becken lokalisiert und 33\% wiesen jedoch distante Läsionen auf [23].
Insgesamt konnte der Nutzen der PSMA-PET/CT in einer Vielzahl von Studien bei Patienten mit BCR nach radikaler Prostatektomie oder perkutaner Bestrahlung (RT) bewiesen werden. In einer Metaanalyse mit Einschluss von 1309 Patienten betrug die Gesamtdetektionsrate $76 \%$. Abhängig vom PSAWert stieg die Detektionsrate von $42 \%$ für PSA-Werte $<0,2 \mathrm{ng} / \mathrm{ml}$, $58 \%$ für 


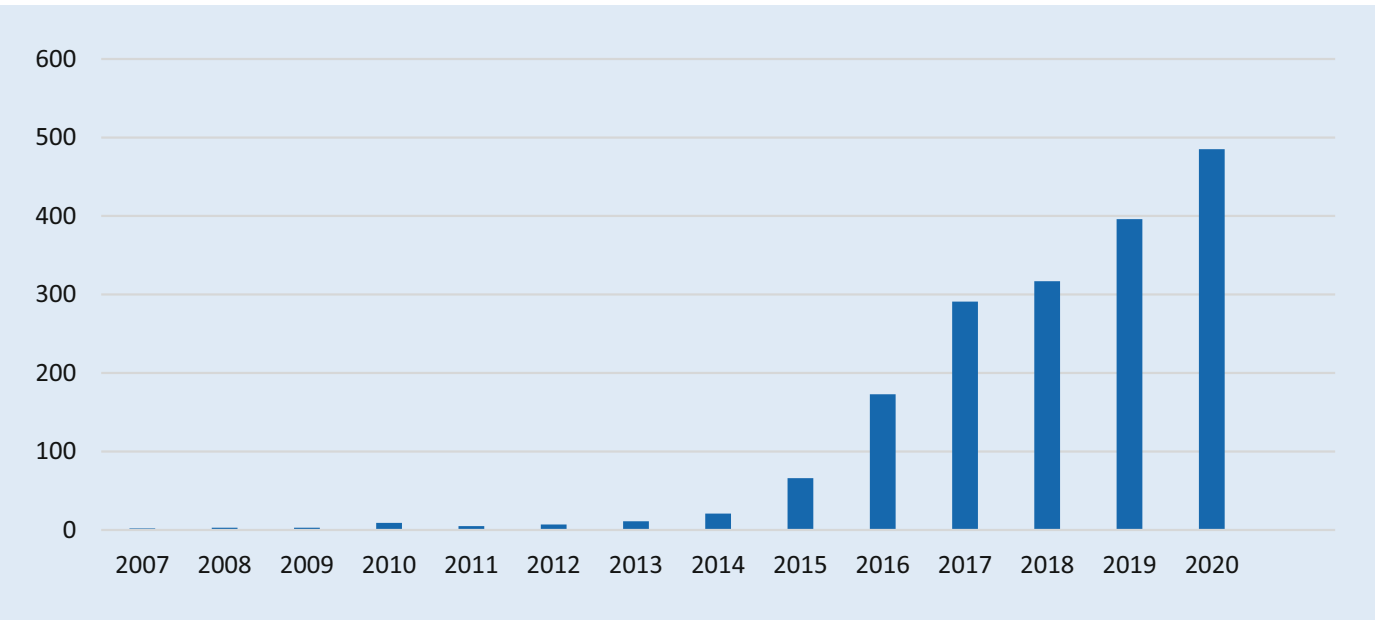

Abb. $2 \triangleleft$ Darstellung der Häufigkeit der Publikationen "PSMA PET prostate cancer" der Plattform PubMed im Zeitraum 2007 bis 2020. (x-Achse: Jahr; $y$-Achse: Anzahl der Publikationen)

Werte von $0,2-1 \mathrm{ng} / \mathrm{ml}, 76 \%$ zwischen einem PSA von $1,0-2,0$ auf $95 \%$ für $\mathrm{Pa}$ tienten mit einem PSA $>2,0 \mathrm{ng} / \mathrm{ml}$. Für die patientenbasierte Analyse betrugen die zusammengefasste Sensitivität und Spezifität jeweils $86 \%$ [24].

Basierend auf den Ergebnissen der PSMA-PET-Bildgebung kommt es in einer beachtlichen Anzahl der Fälle zu Änderungen in der Strahlentherapieplanung. In einer Studie hatten $71 \%$ der eingeschlossenen 129 Patienten vor Strahlentherapie bei primärem, persistierendem oder rezidivierendem Prostatakrebs mindestens eine relevante PSMA-positive Läsion. Bezüglich der anatomischen Verteilung hatten $42 \%$ der Patienten mit BCR pelvine und distante LK-Metastasen. Insbesondere die Detektion von Rezidiven außerhalb des Prostatabetts mit $22 \%$ pelvinen LK- und $4 \%$ distanten LK-Metastasen änderte die geplante Strahlentherapieplanung. Bei $57 \%$ der Patienten wurde die Bestrahlungsplanung durch die Ergebnisse des PSMA-PET modifiziert [25]. Leitliniengerecht empfiehlt die European Association of Urology (EAU) den Einsatz der PSMA-PET/CT bei einem persistierenden PSA $>0,2 \mathrm{ng} / \mathrm{ml}$ zum Metastasenausschluss [26].

Im biochemischen Rezidiv konnte in einer multizentrischen Studie mit $323 \mathrm{~Pa}$ tienten gezeigt werden, dass $62 \%$ sich die Behandlungsstrategie im Vergleich zum konventionellen Staging änderte. Diese signifikante Reduktion der unklaren Rezidivlokalisation wurde durch die PSMAPET erreicht (77\% vor PSMA-PET un- klar vs. 19\% nach PSMA-PET). Es war ein signifikanter Anstieg einer Metastasierung $\mathrm{zu}$ verzeichnen (11\% vor vs. $57 \%$ nach PSMA-PET) getrennt nach oligometastatischer Erkrankung (10\% vor PSMA-PET vs. $38 \%$ danach) und polymetastatischer Situation (1\% vs. 19\%; [27]).

Der zusätzliche Informationsgewinn führte zu der EAU-Empfehlung, die PSMA-PET bei einem PSA $>0,2 \mathrm{ng} / \mathrm{ml}$ nach radikaler Prostataektomie anzubieten, wenn durch die Befunde die nachfolgende Behandlungsentscheidung beeinflusst wird [26]. In der S3-Leilinie gibt es diese Empfehlung zur Beurteilung der Tumorausdehnung, falls sich daraus eine Therapiekonsequenz ergibt [16]. In den letzten Jahren nahm die SalvageLymphadenektomie nach radikaler Prostatektomie zu. In einer retrospektiven Studie betrug der mediane Kurzachsendurchmesser der PSMA-positiven LK $5,8 \mathrm{~mm}$. Von diesen PSMA-positiven LK waren $78 \%$ anhand der morphologischen Kriterien im CT unauffällig mit einem Diameter $<8 \mathrm{~mm}$. Eine Änderung von N0 zu N1 fand in $67 \%$ der Patienten statt ([28]; Fallbeispiel in • Abb. 5).

\section{Staging im oligometastatischen Erkrankungsstadium}

In den letzten Jahren rückt zunehmend auch die Behandlung der Oligometastasierung in den Fokus, einem Krankheitszustand zwischen dem lokalisierten Stadium und der disseminierten systemischen Tumoraussaat mit weniger oder maximal gleich 5 Metastasen [29]. Durch den Paradigmenwechsel in der Therapie kann bei Tumorkontrolle des Primarius und chirurgischer oder strahlentherapeutischer Ausschaltung der Metastasen ein längeres krankheitsfreies Intervall oder möglicherweise Heilung erreicht werden. Dieser Ansatz wird durch eine retrospektive PSMA-PET/CT-Studie mit 319 Patienten untermauert, in der das PSMA-PET/CT in $83 \%$ der Patienten tumorsuspekte Läsionen im biochemischen Rezidiv detektierte. Bei insgesamt 116 Patienten waren Follow-up-Informationen verfügbar, 50 von den 116 Patienten erhielten eine lokale Behandlung (27 externe Bestrahlungen, 19 Operationen, 4 Behandlung mit hochintensivem fokussiertem Ultraschall), und 34 Patienten erhielten eine PSMA-RadioligandenTherapie (RLT). Alle anderen Patienten wurden mittels ADT und/oder Chemotherapie therapiert [30].

In einer retrospektiven Studie an 17 Patienten mit einem nodal oligometastatischen Prostatakarzinom mit BCR konnte die präoperative PSMA-PET/CT eine Sensitivität von $94 \%$, eine Spezifität von $99 \%$ und einen positiven/negativen prädiktiven Wert von 89\%/99,5\% erreichen. Nach der Lymphknotendissektion erhielten $47 \%$ keine weitere Therapie, und bei insgesamt $82 \%$ blieb der PSAWert in der Nachsorge unter $0,2 \mathrm{ng} / \mathrm{ml}$. In dieser Studie zeigte sich, dass die PSMA-PET/CT die Genauigkeit der Detektion von Mikrometastasen verbessern könnte [31]. 
Tab. 1 Physiologische Anreicherung der PSMA-Radioliganden

Biodistribution von PSMA-Liganden im 68Ga-PSMA-PET/CT

Spezifische und unspezifische Speicherung

Intensiv

Tränen- und Speicheldrüsen

Nieren

Darmtrakt v.a. des Dünndarms

Moderat

Leber

Milz

Gering

Ganglien vegetatives Nervensystem

Nasale und ösophageale Mukosa

Stimmbänder

Gallenblase und Gallentrakt

Trachea und proximale Bronchien

Lymphknoten mediastinal, axillär und inguinal

Ausscheidung

Nieren und ableitende Harnwege

\section{Staging im fortgeschrittenen}

polytopen und disseminiert metastasierten Erkrankungsstadium

Ein weiteres Anwendungsgebiet der PSMA-PET/CT ist die Bildgebung des fortgeschrittenen Prostatakarzinoms. Bei Eintritt der Kastrationsresistenz wird normalerweise mittels kontrastverstärkter CT und der Knochenszintigraphie zwischen nichtmetastasiertem (nmCRPC) und metastasiertem kastrationsresistentem Prostatakarzinom (mCRPC) unterschieden, da sich die Behandlungsstrategie in beiden Erkrankungsstadien unterscheidet. In einer retrospektiven Studie an 200 Patienten mit nmCRPC in der konventionellen Bildgebung war die PSMA-PET/CT in 196 Fällen positiv; davon hatten $55 \%$ eine distante Metastasierung [32].

\section{PSMA-PET-geführte Strahlen- therapie}

An 100 Patienten mit BCR nach RP und/oder RT konnten in $76 \%$ der Patienten mit einem medianen PSA-Wert von $1,0 \mathrm{ng} / \mathrm{ml}$ tumorsuspekte Läsionen mit erhöhter PSMA-Expression aufge- zeigt werden. Aufgrund der gewonnenen Erkenntnisse durch die PSMA-PET wurde die ursprüngliche geplante Bestrahlungsplanung in 59\% aller Fälle angepasst. Ein zusätzlicher simultaner integrierter Boost auf das Prostatabett oder die pelvinen LK wurde in $32 \%$ bzw. $63 \%$ appliziert [33]. Die PSMA-PET/CT änderte in einer prospektiven Studie mit 300 eingeschlossenen Patienten das Therapieregime der geplanten SalvageBestrahlung in 29\% der Fälle [34].

Insbesondere in der Strahlentherapie ist man von einer präzisen Tumorbildgebung abhängig, da sie die Basis der Zielvolumendefinition ist. Im Primärstaging wie im Rezidiv ist oftmals keine histopathologische Korrelation möglich, z. B. bei kleinen LK-Metastasen oder Knochenmetastasen. Die hochsensitive und sehr spezifische PSMA-Bildgebung zeigte neben der bereits erwähnten Änderung des Therapieregimes den enormen Informationszugewinn der PET/CT bei niedrigen PSA-Werten. In einer Studie an $1007 \mathrm{~Pa}-$ tienten wurde bei 801 (79,5\%) Patienten mindestens eine rezidivcharakteristische Läsion nachgewiesen. In den Untergruppen mit einem PSA-Bereich $\leq 0,2 \mathrm{ng} / \mathrm{ml}$ und in der Gruppe $0,21 \leq 0,5 \mathrm{ng} / \mathrm{ml}$ betrug die Detektionsrate jeweils noch $46 \%$ [35]. Der gezielte Einsatz der PSMABildgebung v.a. im biochemischen Rezidiv bei Hochrisikopatienten führt $\mathrm{zu}$ einer sehr präzisen Strahlentherapieplanung mit Reduzierung des Risikos möglicherweise nicht erkannter Metastasen, die konsekutiv nicht in das Bestrahlungsfeld eingeschlossen worden wären. Weitere Studien mit prospektivem Design sind jedoch notwendig, um den bereits erkannten Nutzen zu untermauern und zu zeigen, dass sich das Gesamtüberleben der Patienten durch den Einsatz dieser Bildgebung erhöht und die Tumorkontrolle verbessert. Wichtig ist noch hervorzuheben, dass eine PSMA-PET/CT ohne pathologischen Befund die Einleitung einer Salvage-Radiatio nach radikaler Prostatektomie nicht verzögern sollte, da bei niedrigen PSA-Werten $<0,5 \mathrm{ng} / \mathrm{ml}$ die höchsten Erfolgsraten zu erwarten sind [16]

\section{Strukturierte Befundung}

Die PSMA-PET-Bildgebung hat eine bisher nie dagewesene Genauigkeit der Ganzkörperbildgebung in klinischen Studien und in der klinischen Anwendung weltweit gezeigt. Eine standardisierte Interpretation und Befundung mit gemeinsamer Sprache ist für den Befundbericht und in der Kommunikation mit den klinischen Kollegen essenziell, um den maximalen Benefit aus dieser Untersuchungsmethode zu ziehen. Die Klassifizierung nach RECIST ist in der molekularen Bildgebung nicht optimal anwendbar. Eine Methode könnte die strukturierte Befundung und Beschreibung mittels eines molekularen TNMbasierten Berichts sein (Prostate Cancer Molecular Imaging Standardized Evaluation; [36]). Urologen sind bereits schon mit der Einteilung nach der PI-RADS Klassifikation (The Prostate Imaging Reporting and Data System) vertraut [37]. Eine weitere vielversprechende Klassifizierung ist die Einteilung der Läsionen anhand ihrer PSMA-Expression in PSMA-RADS 1-5 und damit in der Wahrscheinlichkeit des Vorliegens eines Prostatakarzinoms [38].

\section{PSMA-PET/MRT}

Die kombinierte Nutzung einer simultanen PET/MRT bietet die Möglichkeit, zusätzlich zu der Aussage der PSMAExpression den hervorragenden Weichteilkontrast der MRT zu kombinieren und weitere funktionelle Parameter wie die Diffusionswichtung zu messen. Diese Informationen haben das Potenzial, die Tumordetektion z.B. im Rahmen einer bildfusionsgestützten Biopsie und das Staging deutlich zu verbessern. Dieses Potenzial wurde bereits 2016 in einer Studie an 66 Patienten mit histologisch gesichertem Prostatakarzinom in der Primärdiagnostik beschrieben. Die simultane PET/MRT zeigte eine statistisch signifikant bessere Tumorlokalisation (AUC 0,88) im Vergleich zur multiparametrischen MRT (AUC 0,73) und der PET-Bildgebung alleine $(0,83$; [17]). In einer weiteren Studie detektierte die PSMA-PET/MRT das Prostatakarzinom in $97,5 \%$ korrekt. Die diagnostische 


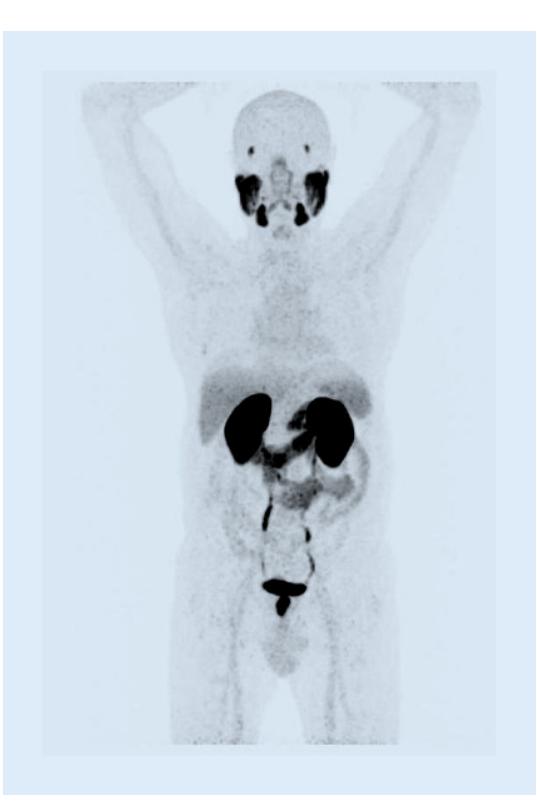

Abb. $3 \triangle$ Maximumintensitätsprojektion (MIP) eines Patienten mit physiologischer Verteilung von Ga68-PSMA-Liganden. Anreicherungen in den Tränen- und Speicheldrüsen, Nasenschleimhaut, Leber, Milz, Nieren, Darm und Harnblase. Bei diesen Patienten Z. n. TUR-P mit erweiterter Pars prostatica urethra und prominenten Ureteren

Genauigkeit stieg mit zunehmendem T-Stadium bis auf $94 \%$ bei T3b an und betrug beim N1-Stadium $93 \%$. In 29\% der Fälle wurde die Behandlungsstrategie durch die Hybridbildgebung geändert [39].

\section{PSMA-basierte Theranostik}

Das PSMA ist ein exzellentes Beispiel eines Theranostikums. In der PET/CT werden die pathologischen Läsionen durch die zielgerichtete Rezeptordarstellung abgebildet, therapeutisch werden diese Rezeptoren mit Beta- oder Alphastrahlen anvisiert und die Tumormanifestationen im Rahmen der PSMARadioliganden-Therapie (PSMA-RLT) bestrahlt (• Abb.6). Die PET/CT findet dabei Einsatz bei der Indikationsstellung zur Radioligandentherapie wie auch in der Beurteilung des Therapieansprechens seine Anwendung.

\section{PSMA-RLT}

Die PSMA-RLT hat sich in den letzten Jahren $\mathrm{zu}$ einem neuen, weiteren Therapieverfahren bei fortgeschrittenen
Erkrankungsstadien und ausgeschöpften konventionellen Therapieverfahren beim mCRPC entwickelt und wird, obwohl sie kein offiziell zugelassenes Therapieverfahren ist, bereits in der „Interdisziplinären Leitlinie der Qualität S3 zur Früherkennung, Diagnose und Therapie des Prostatakarzinoms" des Leitlinienprogramms Onkologie Stand Mai 2019 seit 2018 unter der evidenzbasierten Empfehlung 7.45 als Therapie genannt und unter bestimmten Voraussetzungen empfohlen [16].

\section{Wirkprinzip der PSMA-RLT}

Angelehnt an bereits in der Nuklearmedizin länger etablierte Therapieverfahren, werden hierfür PSMA-Liganden mit radioaktiven Nukliden gekoppelt, die im Gegensatz zu den diagnostischen $\mathrm{Nu}$ kliden für die PSMA-PET-Bildgebung (Beta-Plus-Strahler), Beta-Minus-Strahler, vor allem 177Lutetium (177Lu), oder Alphastrahler, vor allem 225Actinium (22Ac), sind, die jeweils nur eine geringe Reichweichte von unter $1 \mathrm{~mm}$ in Gewebe haben und dadurch in der Lage sind, Zellen in ihrer direkten Umgebung abzutöten. Bei intravenöser Gabe kann durch die Bindung an das PSMA so eine interne Bestrahlung vor allem der Prostatakarzinomzellen und damit eine relativ zielgerichtete Tumortherapie durchgeführt werden bei vergleichsweise wenig Nebenwirkungen. Inzwischen liegen hierzu bereits aus verschiedenen Studien entsprechende Daten vor. Um gesunde Gewebe zu schützen und eine genügend hohe Dosis in den Tumormanifestationen $\mathrm{zu}$ erreichen, wird die Gabe der radioaktiven Substanzen auf mehrere Therapiezyklen verteilt.

\section{Die PSMA-RLT als individueller Heilversuch}

\section{Indikationen und Kontra- indikationen}

Da die PSMA-Therapie bisher kein Routineverfahren ist, gilt sie als palliative Therapie und individueller Heilversuch [32, 40]. Aus diesem Grund muss patientenindividuell die Indikation zur PSMA-Therapie gestellt werden. Gemäß den Richtlinien der europäischen Gesellschaft für Nuklearmedizin (EANM) zur Radionuklidtherapie mit $177 \mathrm{Lu}-$ markierten PSMA-Liganden sowie den Empfehlungen der S3-Leitinie zum Prostatakarzinom sollte diese im Rahmen eines interdisziplinären Tumorboards (mindestens ein Urologe/Onkologe, $\mathrm{Nu}$ klearmediziner und Radioonkologe) als ausgesprochene Konsensus-Empfehlung gestellt werden, wobei der Patient im Vorfeld über den experimentellen Charakter der Intervention ausdrücklich aufgeklärt werden muss [16, 32, 40]. Für die Patientenauswahl haben sich einige Einschlusskriterien etabliert, die ebenfalls in den Richtlinien der EANM festgehalten sind. Dazu zählen neben dem histologisch gesicherten Befund eines Prostatakarzinoms eine nichtresektable Metastasierung, eine im Verlauf gesicherte Kastrationsresistenz sowie ein (erneuter) Tumorprogress unter leitliniengerechter Therapie sowie die Ausschöpfung aller leitliniengerechten Therapien bzw. der beim Patienten möglichen Therapien (z.B. Patient ist „unfit for chemotherapy“). Zu diesen zählen die sekundären Hormontherapien mit Abirateron und Enzalutamid sowie Chemotherapien mit Docetaxel oder Cabazitaxel sowie eine Therapie mit 223Radiumdichlorid (Alpharadin, Xofigo $^{\circledR}$ ), ein weiteres nuklearmedizinisches Therapieverfahren mittels eines Alphastrahlers, das bei ausschließlicher osteoplastischer Skelettmetastasierung ohne viszerale Metastasen angewendet werden darf. Für die PSMA-RLT wird teils ein Abstand von etwa 6 Wochen zur letzten myelosuppressiven Therapie empfohlen, insbesondere bei auffälligen Blutbildwerten [32]. Vor einer PSMATherapie muss zudem durch eine Diagnostik mittels PSMA-Liganden, vorzugsweise einer PSMA-PET, eine ausreichende PSMA-Expression der Tumorläsionen nachgewiesen werden, wobei es für diese keine harten Cut-off-Werte gibt. In Anlehnung an andere Theranostika sollte der Uptake mindestens oberhalb der Organe mit physiologischem Uptake liegen, beispielsweise der Leber [40]. Sowohl die EANM als auch die S3-Leitlinie zum Prostatakarzinom raten $\mathrm{zu}$ einer Durchführung der PSMA-Therapie im 


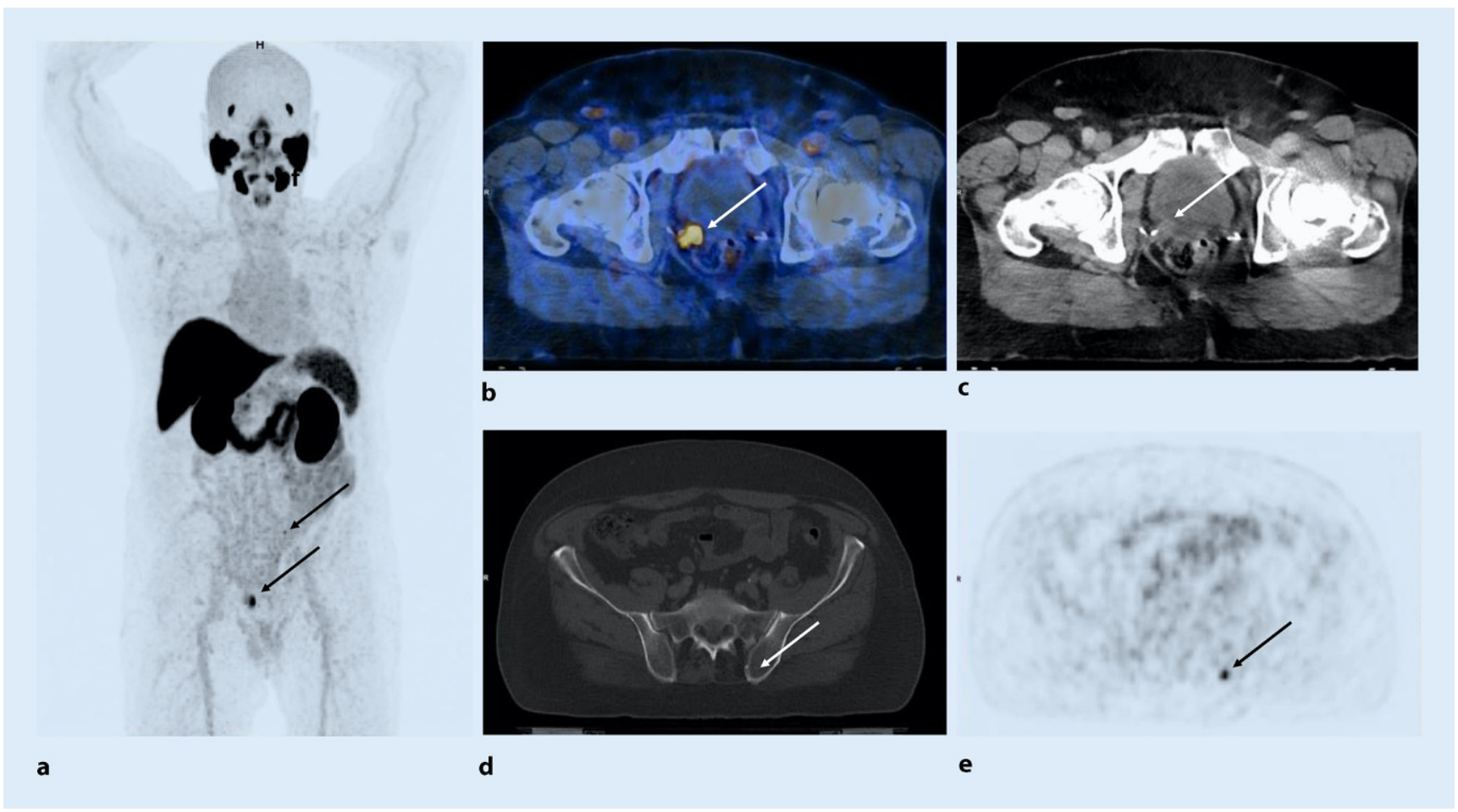

Abb. 4 A Fallbeispiel: Rezidivdiagnostik mittels F18-PSMA-1007 PET/CT bei einem 69-jährigen Patienten mit PSA-Anstieg 14 Jahre nach radikaler Prostatektomie (Gleason-Score: 7a; pT2c pN0 cM0, PSA-Nadir: 0,08 ng/ml). Aktueller PSA-Wert: $1,0 \mathrm{ng} / \mathrm{ml}$. Die PSMA-PET/CT zeigt ein Lokalrezidiv an der ehemaligen Prostatabasis rechts/Samenblase (Pfeile a-c). Zusätzlich erhöhte PSMA-Expression im linken Os ilium im Sinne einer ossären Filia ohne CT-morphologisches Korrelat (Pfeile a, d, e). Rezidivdiagnostik mittels F18-PSMA-1007 PET/CT. a Maximumintensitätsprojektion (MIP). b Fusioniertes PET/CT transversal. c CT transversal im Weichteilfenster, Pfeil ehemalige Prostataloge rechts. d CT im Knochenfenster transversal ohne metastasensuspekte Mehrsklerosierung. e PET transversal mit fokaler PSMA-Expression im linken Os ilium

Rahmen von prospektiven Studien [16, $32,40]$.

Als potenzielle Kontraindikationen, bei welchen eine PMSA-Therapie nicht durchgeführt werden sollte, benennt die Richtlinie der EANM eine Lebenserwartung unter 6 Monaten (ECOG Performance-Status $>2$ ), sofern diese nicht maßgeblich durch das Leiden an den Tumorsymptomen bestimmt wird. Des Weiteren sollten keine medizinischen oder Strahlenschutz-Sicherheitsrisiken gegen eine Isolierung auf einer nuklearmedizinischen Therapiestation bestehen, die oft durch nationale Gesetze gefordert wird. Aufgrund der renalen Elimination der PSMA-Liganden sind außerdem höchstgradige Obstruktionen des Nieren- und Harntrakts oder eine Hydronephrose Kontraindikationen. Bei Patienten mit einem erhöhten Risiko für Harnretentionen wird empfohlen, als Basisuntersuchung vor einer potenziellen PSMA-RLT eine Nierenszintigraphie (mit 99mTc-MAG3 oder 99mTc-DTPA) durchzuführen. Die Nieren- und Leberfunktion sollte laborchemisch überprüft werden, wobei eine $\mathrm{GFR}<30 \mathrm{ml} / \mathrm{min}$ oder ein Serumkreatinin-Wert größer als das 2-Fache des oberen Normbereichs sowie Leberenzyme größer das 5-Fache des oberen Normbereichs weitere Ausschlusskriterien sind. Überdies sollte eine Myelosuppression laborchemisch ausgeschlossen werden, insbesondere eine Leukozytenzahl unter $2,5 \times 10^{9} / 1$ sowie eine Thrombozytenzahl $<75 \times 10^{9} / 1$. Zusätzlich wird darauf hingewiesen, dass bei Patienten, bei welchen absehbar zeitnahe andere Interventionen erforderlich sein werden, wie eine Bestrahlung oder eine Operation, z. B. bei Affektion des Rückenmarks oder instabilen Frakturen, je nach Allgemeinzustand zuerst eine Nutzen-Risiko-Abwägung erfolgen und die PSMA-RLT ggf. später durchgeführt werden sollte. Eine weitere Kontraindikation stellt auch eine nicht ausreichende PSMA-Expression in der PSMA-Bildgebung dar, wobei insbeson- dere PSMA-negative Lebermetastasen ein Ausschlusskriterium sind, auch wenn möglicherweise weitere Tumormanifestationen noch eine ausreichende PSMAExpression aufweisen [40].

Da bisher keine wegweisenden Daten für potenzielle Wechselwirkungen mit anderen medizinisch indizierten Begleittherapien vorliegen, kann eine begleitende Hormontherapie oder antiresorptive Therapie bei Knochenmetastasen, eine Antikoagulation sowie eine Gabe von Steroiden erfolgen $[32,40]$.

\section{Therapievorbereitung und -ablauf}

Wurde für einen Patienten mit mCRPC in einem interdisziplinären Tumorboard eine PSMA-RLT empfohlen, muss aus nuklearmedizinscher Sicht eine PSMABildgebung mit ausreichender PSMAExpression sowie ein aktuelles Labor (K, $\mathrm{Ca}$, Kreatinin, LDH, Bilirubin [gesamt], AST, CRP, kleines Blutbild, AP, PSA) vor- 


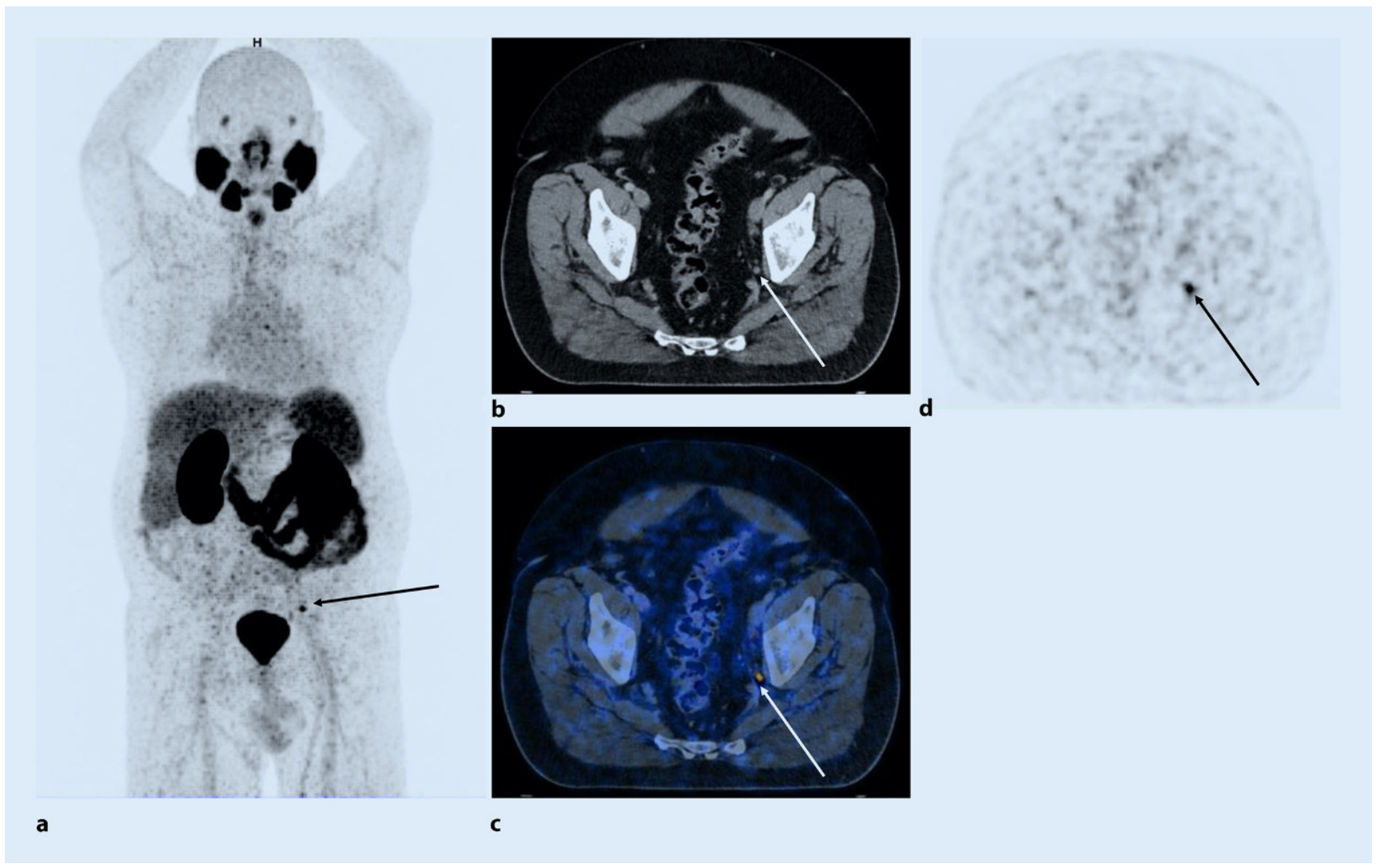

Abb. 5 \ Fallbeispiel: Restaging bei PSA-Persistenz mittels Ga68-PSMAI\&T bei einem 62-jährigen Patienten 1,5 Jahre nach radikaler Prostatektomie (Gleason-Score: 7b; pT2c pNx cM0, PSA-Nadir: 0,4 ng/ml). Aktueller PSA-Wert: 0,6 ng $/ \mathrm{ml}$. Die PSMAPET/CT zeigt eine PSMA-exprimierende LK-Metastase (Pfeile) im A.-iliaca-interna-Gebiet links mit einer Größenausdehnung von $6 \mathrm{~mm}$ in der CT. Restaging bei postoperativer PSA-Persistenz mittels Ga68-PSMAI\&T PET/CT. a MIP. b CT transversal im Weichteilfenster. c Fusionierte PET/CT transversal. d PET transversal mit fokaler PSMA-Expression des metastasensuspekten LK

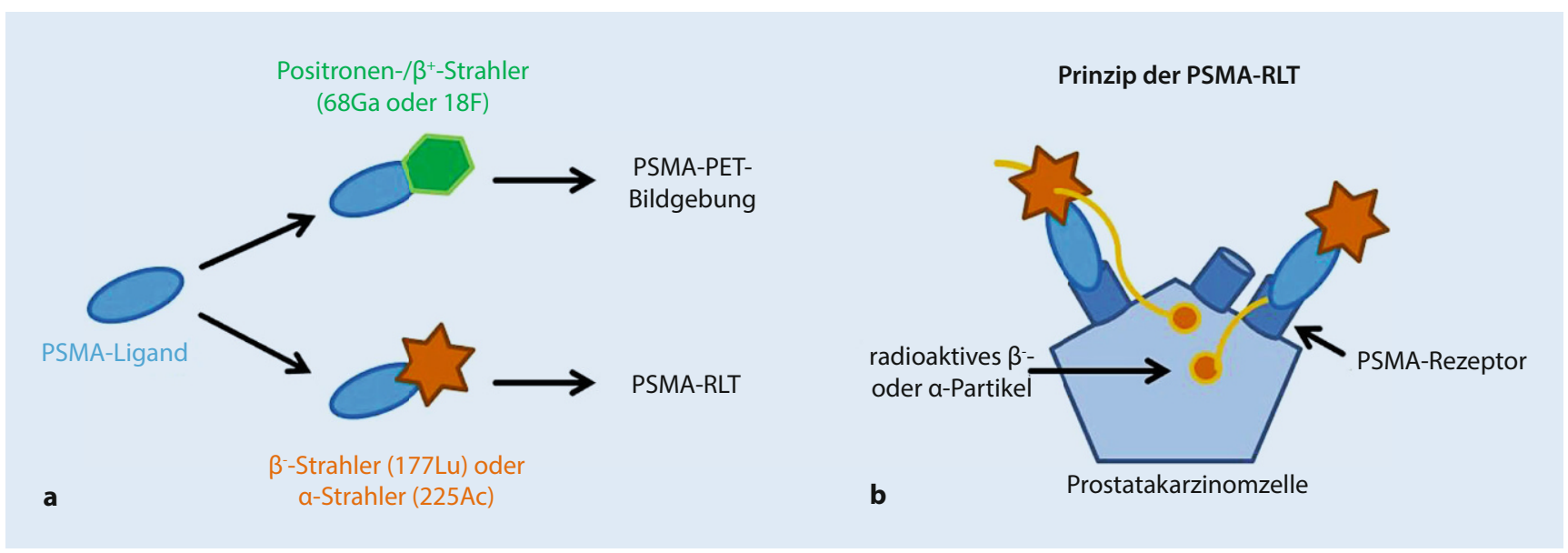

Abb. 6 A a Schematische Darstellung der PSMA-Liganden gekoppelt mit Positronen-/ $\beta^{+}-$Strahlern wie 68Gallium (68Ga) und 18 Fluor (18F) zur PSMA-PET-Bildgebung für PET/CT und PET/MRT sowie gekoppelt mit $\beta$-Strahlern wie 177Lutetium (177Lu) oder $\boldsymbol{a}$-Strahlern wie 225Actinium (225Ac) für eine PSMA-Radioligandentherapie (PSMA-RLT).b Prinzip der PSMA-RLT. Die mit $\beta$ - oder $\alpha$-Strahlern gekoppelten PSMA-Liganden binden an die PSMA-Rezeptoren auf den Prostatakarzinomzellen. Die radioaktiven $\beta$ - oder a-Partikel kommen dadurch in unmittelbare Nähe zu den Prostatakarzinomzellen, welche dadurch bestrahlt und zerstört werden 
liegen. Je nach vorliegenden Befunden sollte fakultativ über die Notwendigkeit einer weiteren Nierenabklärung mittels Nierenszintigraphie oder eine Speicheldrüsenszintigraphie diskutiert werden, da diese ebenfalls eine hohe PSMAExpression aufweisen und somit bei einer PSMA-RLT auch einer erhöhten Strahlenexposition ausgesetzt sind [32, 40]. Der Patient muss mindestens $24 \mathrm{~h}$ vor einem Therapiezyklus von einem Nuklearmediziner umfassend über die Therapie aufgeklärt werden.

In den meisten europäischen Ländern ist für die PSMA-RLT ein mindestens 48stündiger - oder je nach lokalen Abläufen auch ein mehrtägiger - Aufenthalt auf einer nuklearmedizinischen Therapiestation mit Abklinganlage zur Sammlung radioaktiver Ausscheidungen aus Strahlenschutzgründen gesetzlich gefordert. Am Aufnahmetag wird der Patient ärztlich untersucht. Vor der Applikation der PSMA-Liganden sollte eine ausreichende Hydrierung des Patienten unter Berücksichtigung möglicher kardiovaskulärer oder renaler Begleiterkrankungen vorliegen, in der Regel durch die Infusion von etwa 1-2 1 Infusionslösung kurz vor sowie nach der Gabe der PSMA-Liganden. Gegebenenfalls kann eine Kühlung der Speicheldrüsen mittels Kühlkompressen 30 min bis $1 \mathrm{~h}$ vor sowie bis $4 \mathrm{~h}$ nach der Applikation der PSMALiganden zum Schutz der Speicheldrüsen erfolgen, deren Nutzen jedoch noch nicht ausreichend durch Studien belegt ist $[41,42]$. Gegebenenfalls können begleitend eine Antiemese (z. B. durch Ondansetron), die Gabe von Steroiden als Ödemprophylaxe bei Metastasen mit intraspinaler oder intrakranieller Lokalisation sowie die Gabe von Diuretika und Laxanzien zur schnelleren Elimination von ungebundenem 177Lu-PSMA verabreicht werden. Die PSMA-Liganden werden nach entsprechender Vorbereitung des Patienten entweder per Perfusor oder durch langsame direkte Injektion intravenös appliziert. Anschließend sollte der Patient weiterhin auf eine ausreichende Hydrierung achten, welche sowohl oral als auch intravenös erfolgen kann [32, 40].

Je nach Standort werden ggf. im Verlauf des stationären Aufenthalts für dosimetrische Zwecke Blutproben beim Patienten entnommen sowie ein oder mehrere Aufnahmen zur quantitativen Auswertung erstellt, idealweise mittels SPECT/CT, um absorbierte Dosen der Tumorläsionen sowie der Risikoorgane ermitteln zu können. Gegebenenfalls können sich daraus für weitere Therapiezyklen individuellere applizierte Aktivitäten ableiten.

\section{Therapieschema, Nachsorge und potenzielle Neben- wirkungen}

In der Literatur werden basierend auf den publizierten Studiendaten pro Therapiezyklus Standardaktivitäten an 177Lu zwischen 6 und 7,4 GBq angegeben sowie ein Standardabstand von etwa 6 bis 8 Wochen zwischen den einzelnen Therapiezyklen eingehalten und 4 bis 6 Therapiezyklen angestrebt, in Abhängigkeit vom Therapieansprechen, der Prognose und potenziellen Nebenwirkungen am Knochenmark oder den Nieren [32, 40, 43-50]. Vor allem bei Patienten mit einer Lebenserwartung von mehr als 1 Jahr wird empfohlen, an den Nieren eine kumulativ absorbierte Dosis von mehr als 40 Gy nicht zu überschreiten. Bereits bei jeder Therapie sollte durch die Kontrolle des PSAWerts und posttherapeutische Ganzkörperszintigraphien ggf. mittels SPECT/CT ein Response-Assessment durchgeführt werden. Zusätzlich sollte in regelmäßigen Abständen, idealerweise bereits nach 2 Zyklen, ein erneutes Restaging zur Therapieevaluation erfolgen, bevorzugt mittels PSMA-PET sowie ggf. auch mittels einer zweiten Modalität, um PSMA-negative Tumormanifestationen auszuschließen bzw. detektieren zu können, wozu sich Hybridverfahren wie die PET/CT oder PET/MRT mittels PSMA-Liganden hervorragend eignen [40].

Zur Nachsorge zwischen den Therapiezyklen wird empfohlen, dass alle 2 bis 3 Wochen in Abhängigkeit von den Ausgangsbefunden bis 12 Wochen nach dem letzten Therapiezyklus regelmäßige Laborkontrollen vor allem der Blutbild-, Nieren- und ggf. Leberwerte erfolgen sollten, welche bspw. beim behandelnden Onkologen durchgeführt werden können. (Ein schematischer Ablauf der
PSMA-RLT ist in - Abb. 7 dargestellt.) Bei einer Kontrolle des PSA-Werts sollte auf einen ausreichenden Abstand zur PSMA-Therapie geachtet werden - meist etwa 4 Wochen -, da direkt im Anschluss an die PSMA-Therapie aufgrund von therapieinduziertem Zellzerfall ein falsch $\mathrm{zu}$ hoher PSA-Wert detektiert werden und als Progress fehlinterpretiert werden könnte. Bei sehr gutem Ansprechen oder beim Auftreten von Nebenwirkungen kann die Therapie auch zwischenzeitlich pausiert und bei erneutem Progress oder nach Rekonstitution des Patienten wiederaufgenommen werden.

Insgesamt gilt die PSMA-RLT als eine sehr sichere und nebenwirkungsarme Therapie. Hämatotoxizitäten Grad 3-4 traten je nach Studie bei weniger als $10 \%$ der Fälle auf und auch Grad-3/4-Toxizitäten an anderen Organen, auch an den Speicheldrüsen, wurden nur in etwa $5 \%$ der Fälle gefunden [42, 47, 48, 51-53].

\section{Wirksamkeit der PSMA-RLT und Erfolgsaussichten}

Als Therapieerfolg wird ein biochemisches Ansprechen mit einem rückläufigen PSA-Wert von $50 \%$ oder mehr nach mehreren Therapiezyklen gewertet sowie ein Ansprechen in der PSMA-Bildgebung [48]. In Studien konnte ein deutlicher PSA-Rückgang von teils mehr als $50 \%$ bei über der Hälfte der Patienten beobachtet werden, und mehr als $75 \%$ der Patienten erreichte zumindest eine partielle Response ([52-55]; Fallbeispiel in

- Abb. 8). Tumorassoziierte Schmerzen konnten in mehr als der Hälfte der Patienten deutlich vermindert werden mit einer gesteigerten Lebensqualität $[49,50$, $56,57]$.

\section{Eigentlich bestehen Kontra- indikationen beim Patienten für eine PSMA-RLT ...}

Einige Patienten mit mCRPC, die nach ausgeschöpften leitliniengerechten Therapien vom Tumorboard eine Empfehlung zur 177Lu-PSMA-Therapie bekommen, erfüllen teils die genannten Indikationen nach bereits mehrfachen Vortherapien nicht bzw. weisen Kontraindikationen auf. Würden die Patienten 


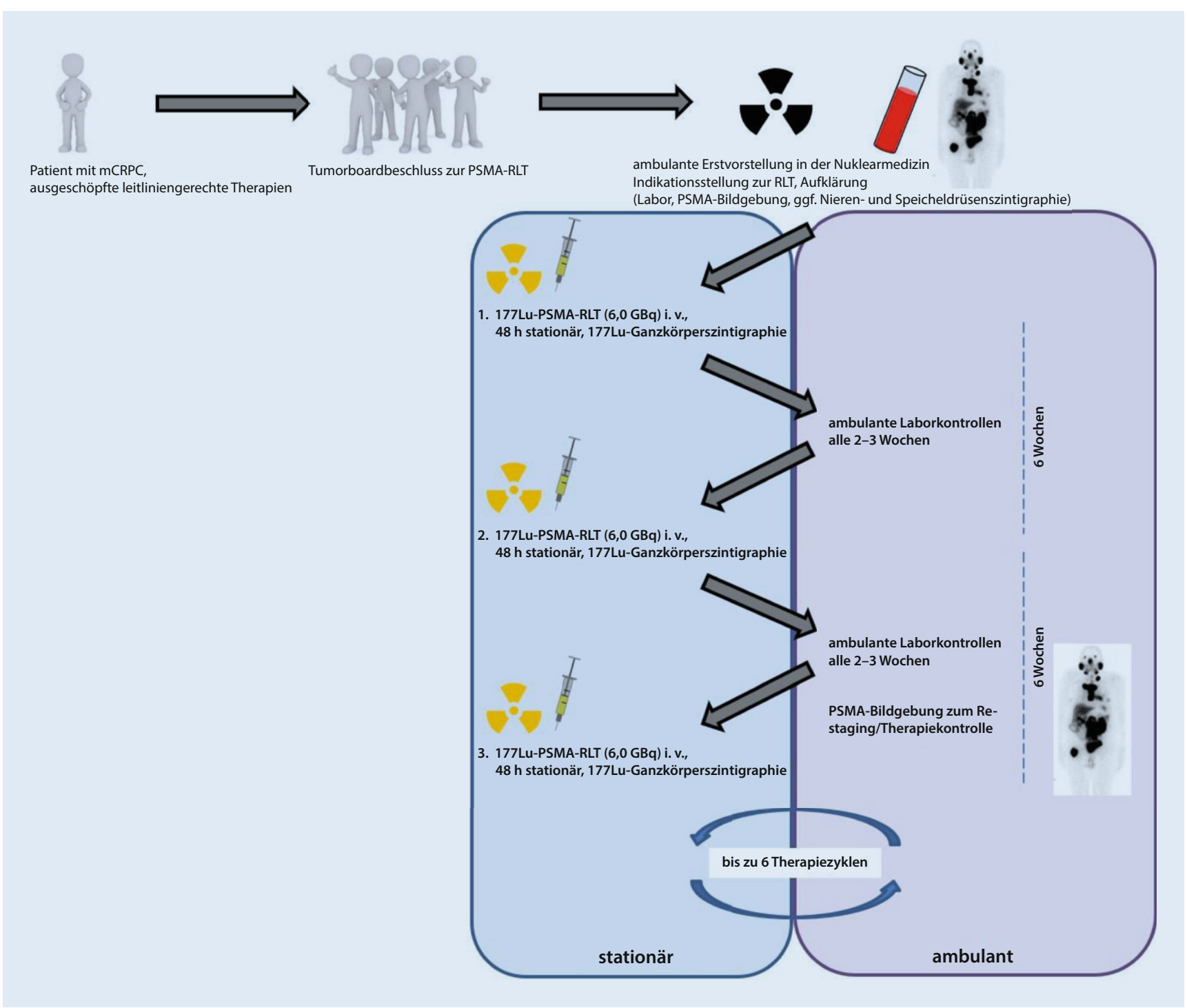

Abb. 7 ॥ Schematischer Ablauf einer PSMA-Radioligandentherapie (PSMA-RLT) mit 177Lutetium (177Lu) nach dem Ulmer Konzept. Der Patient mit metastasiertem kastrationsresistentem Prostatakarzinom (mCRPC) wird nach Ausschöpfen der leitliniengerechten Therapien bei weiterer Krankheitsprogression vom Urologen im interdisziplinären Tumorboard zur Besprechung weiterer Therapien vorgestellt. Bei einem Tumorboardbeschluss zur PSMA-RLT stellt sich der Patient anschließend ambulant in der Nuklearmedizin vor. Dort wird die Indikation zur RLT gestellt und der Patient umfassend aufgeklärt. Zur Indikationsstellung müssen ein aktuelles Labor sowie eine PSMA-Bildgebung (PSMA-PET/CT oder-MRT) mit dem Nachweis einer ausreichenden PSMA-Expression der Tumormanifestationen vorliegen respektive durchgeführt werden. Der Nuklearmediziner entscheidet in Abhängigkeit von Anamnese und Labor über die Notwendigkeit einer zusätzlichen Nieren- und/oder Speicheldrüsenszintigraphie beim Patienten. Danach kann der stationäre Aufenthaltzur 177Lu-PSMA-RLT geplant werden. DerPatient bleibt dabei aus Strahlenschutzgründen $48 \mathrm{~h}$ stationär, bekommt am Aufnahmetag die Standardaktivität von 6,0 GBq 177Lu intravenös appliziert und erhält am zweiten stationären Tag eine 177Lu-Ganzkörperszintigraphie zur Therapieevaluation und -kontrolle. Nach der Entlassung werden ambulant alle 2 bis 3 Wochen in Abhängigkeit von den Ausgangswerten Laborkontrollen durchgeführt und mit der Nuklearmedizin kommuniziert. Nach 6 Wochen erfolgt der 2 . Therapiezyklus. Kurz vor dem 3. Therapiezyklus erfolgt eine erneute PSMA-Bildgebung (PSMA-PET/CT oder-MRT) zum Restaging/zur Therapiekontrolle. Nach Ausschluss eines Progresses kann dann der 3. Therapiezyklus stattfinden. Insgesamt werden bis zu 6 Therapiezyklen angestrebt, wobei erneut kurz vor dem 5 . und nach dem 6. Therapiezyklus Kontrolluntersuchungen mittels PSMA-Bildgebung (PSMA-PET/CT oder-MRT) erfolgen 


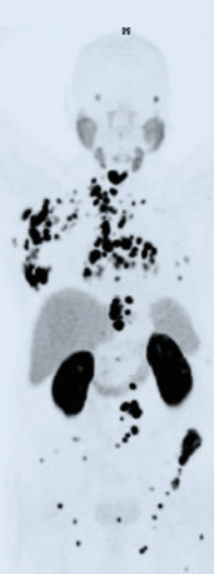

12/18 PSMA-PET Staging vor 177Lu-PSMA-RLT, PSA-Wert $9,41 \mathrm{ng} / \mathrm{ml}$

a

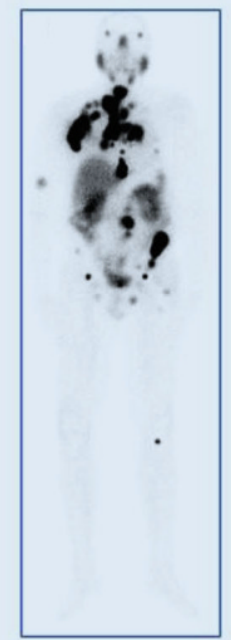

01/19 1. 177Lu-PSMARLT (6.307 MBq), e PSA-Wert $8,56 \mathrm{ng} / \mathrm{ml}$

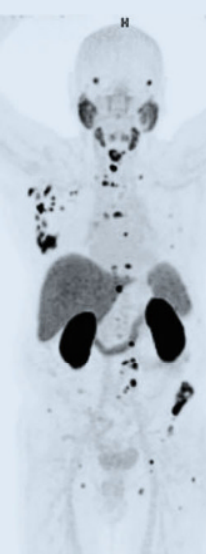

04/19 PSMA-PET Restaging nach 2x 177Lu-PSMA-RLT,

b

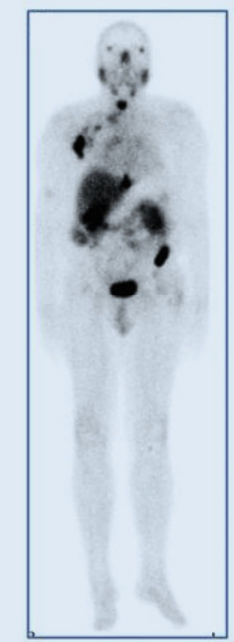

02/19 2. 177Lu-PSMARLT (6.036 MBq), f

g
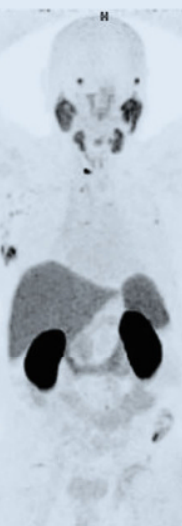

09/19 PSMA-PET Restaging nach 4x 177Lu-PSMA-RLT PSA-Wert 0,44 ng/ml

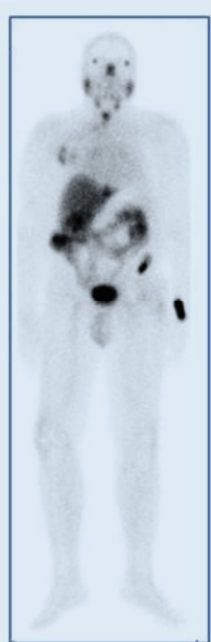

04/19 3. 177Lu-PSMA RLT $(6.036 \mathrm{MBq})$ PSA-Wert $1,47 \mathrm{ng} / \mathrm{ml}$

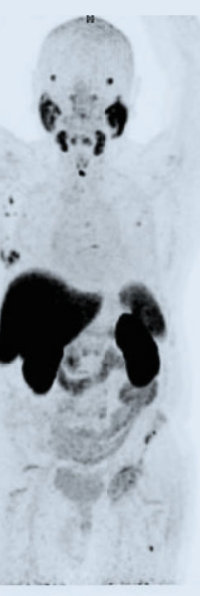

01/20 PSMA-PET Restaging nach 5x 177Lu-PSMA-RLT,

d
PSA-Wert 0,27 ng/ml

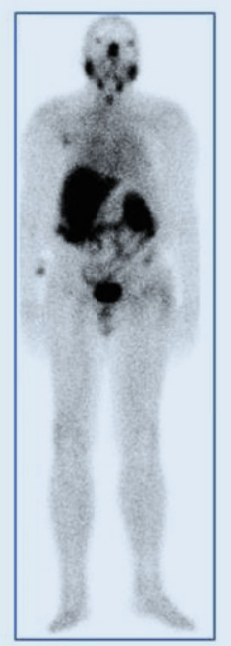

10/19 5. 177Lu-PSMA RLT (4.610 MBq), PSA-Wert 0,28 ng/ml

Abb. 8 A Fallbeispiel: 79-jähriger Patient mit initial ossär metastasiertem Prostatakarzinom, cT1c, pTx, pNx, cM1 (ossär), Gleason-Score $(4+4)=8$, Erstdiagnose 11/2006. Primär kurative Radiatio der Prostata und Lymphabflusswege, parallel Therapiebeginn mit LHRH-Analogon und Osteoprotektion bis 02/2016. Bei Progress Wechsel auf Bicalutamid bis 07/2016. Therapiebeginn mit Abirateron bei erneutem Progress; bei Komplikationen im Verlauf pausiert und bei szintigraphischem ossärem Progress 09/2017 (PSA 3,72 ng/ml) wieder begonnen. 09/2017-04/20186 Applikationen von 223Radiumdichlorid (Xofigo ${ }^{\circledR}$ ). Therapiewechsel aufEnzalutamid 06/2018 bei PSA-Anstieg auf 19,7 ng/ml; unregelmäßige Einnahme bei Komplikationen bis zum erneuten PSA-Anstieg auf $43 \mathrm{ng} / \mathrm{ml}$ und Nachweis von Lymphknotenmetastasen 09/2018; dann kontinuierliche Einnahme. Bei fehlender Eignung für eine Chemotherapie Entscheidung zur 177Lu-PSMA-RLT im Tumorboard. In der PSMA-PET (a) intensive PSMA-Expressionen der Lymphknoten- sowie der disseminierten Skelettmetastasen. Es erfolgten 2 Zyklen 177LuPSMA-RLT (e, f) mit guter Aufnahme in die Metastasen. Deutliches PSA-Ansprechen sowie größenregrediente Metastasen in derRestaging-PET/CT 04/2019. Durchführung von zwei weiteren Therapiezyklen (g,h) mit weiterem Rückgang des PSA-Werts und regredienten Metastasen in der Restaging-PET/CT 09/2019. Zur Konsolidierung erfolgte ein weiterer Therapiezyklus mit reduzierter Aktivität 10/2019. In der PET/CT 01/2020 zeigten sich bei konstantem PSA-Wert nur vereinzelte neue Läsionen bei ansonsten gutem Therapieergebnis, sodass ein abwartendes Verhalten als Konsensusempfehlung im Tumorboard ausgesprochen wurde. Patient mit mCRPCunter 177Lu-PSMA-RLT.a Ga-68-PSMA-PET/CT zum Staging-Maximumintensitätsprojektion (MIP). b-d F18-PSMA-PET/CT zum Restaging - Maximumintensitätsprojektion (MIP). e-i Ausschnitte der 177Lu-PSMA-RLTGanzkörperszintigraphien etwa $24 \mathrm{~h}$ nach intravenöser Gabe der 177Lu-PSMA-Radioliganden, jeweilig applizierte Aktivitäten und PSA-Werte zum Therapiezeitpunkt im Bild. (Alle Ansichten rechts - anterior - links) 
nach einer individuellen Nutzen-RisikoAbschätzung jedoch trotzdem von einer PSMA-Therapie profitieren, gibt es die Möglichkeit, diese unter speziellen Bedingungen sowie im Konsens durch Tumorboardbeschluss dennoch durchzuführen. Sollte bspw. durch die myelosuppressiven Vortherapien eine relevante Anämie oder Thrombopenie vorliegen, kann durch Transfusionen ein $\mathrm{Zu}$ stand erreicht werden, in welchem eine PSMA-Therapie durchgeführt werden kann. Eine Steigerung des Hämoglobinwerts ist dabei entweder durch eine Transfusion ein paar Tage vor der Therapie oder nach der Therapie möglich. Eine Gabe von Thrombozyten kann jederzeit auch vor der PSMA-Therapie erfolgen, da diese sehr strahlenresistent sind. Somit sind nach eigener Erfahrung auch Patienten mit initialen Hämoglobinwerten um $6 \mathrm{~g} / \mathrm{l}$ oder mit Thrombozytenwerten von weniger als $50.000 \mathrm{G} / \mathrm{l}$ therapierbar. Auch Patienten mit einer Knochenmarkkarzinose, die per se eine schlechte Prognose darstellt, können so unter regelmäßigen Blutbildkontrollen und ggf. unter Transfusionen therapiert werden. Bei bestehenden relevanten Harnabflussstörungen mit deutlich auffälliger Nierenszintigraphie kann ggf. eine Befundverbesserung und eine Therapiemöglichkeit nach urologischen Interventionen wie einer Harnleiterschienung oder einer perkutanen Nephrostomie erreicht werden, bei welchen die Ausscheidung der PSMA-Liganden gewährleistet ist. Auch bei deutlich eingeschränkter Nierenfunktion mit einer $\mathrm{GFR}<30 \mathrm{ml} / \mathrm{min}$ oder Dialysepflichtigkeit ist im Einzelfall durch eine Dosisanpassung mit Reduktionen der applizierten Aktivität auf 1,5-2 GBq 177LuPSMA-Liganden eine Therapie durchführbar. Bei bisher nicht nachgewiesenen Interaktionen mit anderen Therapien kann eine 177Lu-PSMA-Therapie auch in Kombination mit Hormontherapien oder lokalen Verfahren angewandt werden, wie z.B. einer lokalen Radiatio bei pathologischen Frakturen oder einer Affektion des Spinalkanals, da durch die lokale Radiatio kein wesentlich erhöhtes Risiko für eine erhöhte Toxizität besteht. Auch kann patientenindividuell die Aktivität oder der Abstand zwischen den The- rapien angepasst werden und zur Reduktion von Nebenwirkungen weniger Aktivität in kürzeren Intervallen von bspw. 4 Wochen gegeben werden.

\section{PSMA-RLT mit 177Lu oder 225Ac?}

Nach der PSMA-RLT mit 177Lu hat sich auch die PSMA-Therapie mit 225Ac an einigen Zentren etabliert. Nach den bisherigen Studien ist die PSMA-Therapie mit 225Ac insgesamt toxischer, kann jedoch gute Erfolge aufweisen, teils auch nach bereits durchgeführten Therapiezyklen mit 177Lu. Sie kann daher ggf. empfohlen werden, wenn unter $177 \mathrm{Lu}$ kein Ansprechen erreicht wird bzw. wenn dafür bereits initial die Wahrscheinlichkeit nicht hoch ist [58-60].

\section{Aktuelle Forschung und Ausblick für die Zukunft}

Während aktuelle prospektive Studien u. a. noch bestehende offene Fragen zum generellen Therapieschema der $177 \mathrm{Lu}$ PSMA-RLT wie einem Aktivitätsstandard sowie einem Standardintervall zwischen den Zyklen adressieren, gibt es bereits auch Studien zur weiteren Evaluation der PSMA-Therapien gegenüber den etablierten Therapien, zu Immuntherapien wie Pembrolizumab oder neuartigeren Therapieansätzen wie bspw. PARP-Inhibitoren wie Olaparib oder zu Kombinationstherapien aus mehreren Verfahren. Bei ClinicalTrials.gov können Stand Ende Januar 202119 Studien mit PSMA-Therapien gefunden werden, von welchen in 11 177Lu-PSMA gegen eine etablierte oder in Kombination mit einer etablierten Therapie geprüft werden, 3 Studien, bei welchen 225AcPSMA geprüft wird, sowie 3 Studien mit weiteren Therapieansätzen wie P-PSMA CAR-T-Zellen oder einem Thorium227-markieren Antikörper. Bereits 16 der 19 Studien rekrutieren zurzeit Patienten. Besonders interessant ist dabei die Phase-3-Studie VISION, bei der 177LuPSMA-617 mit oder ohne Abirateron oder Enzalutamid vs. Abirateron oder Enzalutamid allein getestet wird.

Auch von großem Interesse ist die Phase-2-Studie TheraP, bei der nach einem Progress nach einer Chemotherapie mit Docetaxel die 177Lu-PSMA-RLT mit einer Chemotherapie mit Cabazitaxel verglichen wird, wobei bei ersten Zwischenauswertungen die 177Lu-PSMARLT sowohl bei der Ansprechrate des PSA-Werts um $\geq 50 \%$ zum Ausgangswert als auch beim längeren progressionsfreien Überleben deutlich besser abschneidet als die Chemotherapie mit Cabazitaxel.

Für die Zukunft wird es essenziell sein, prognostische und prädiktive Werte $\mathrm{zu}$ identifizieren, um die geeigneten Patienten zu selektionieren, z.B. nach Tumorlast, PSMA-Expression, Knochenmarkinfiltration, Nierenfunktion, Vortherapien oder der Tumorheterogenität sowie dem Stellenwert der FDG-PET zur Identifikation PSMA-negativer, entdifferenzierter Tumormanifestationen, da bspw. in einzelnen Studien bereits gezeigt werden konnte, dass z. B. eine der 177LuRLT vorausgegangene Chemotherapie sich schlechter auf das Gesamtüberleben auswirkt als bei initialer 177Lu-RLT [61].

\section{Korrespondenzadresse}

\section{Dr. med. Michael Grunert}

Klinik für Nuklearmedizin, Bundeswehrkrankenhaus UIm

Oberer Eselsberg 40, 89081 Ulm, Deutschland michael.grunert@uni-ulm.de

Funding. Open Access funding enabled and organized by Projekt DEAL.

\section{Einhaltung ethischer Richtlinien}

Interessenkonflikt. M. Grunert, N. Eberhardt, V. Prasad und A.J. Beer geben an, dass kein Interessenkonflikt besteht.

Für diesen Beitrag wurden von den Autoren keine Studien an Menschen oder Tieren durchgeführt. Für die aufgeführten Studien gelten die jeweils dort angegebenen ethischen Richtlinien.

Open Access. Dieser Artikel wird unter der Creative Commons Namensnennung 4.0 International Lizenz veröffentlicht, welche die Nutzung, Vervielfältigung Bearbeitung, Verbreitung und Wiedergabe in jeglichem Medium und Format erlaubt, sofern Sie den/die ursprünglichen Autor(en) und die Quelle ordnungsgemäß nennen, einen Link zur Creative Commons Lizenz beifügen und angeben, ob Änderungen vorgenommen wurden.

Die in diesem Artikel enthaltenen Bilder und sonstiges Drittmaterial unterliegen ebenfalls der genannten 
Originalien

Creative Commons Lizenz, sofern sich aus der Abbildungslegende nichts anderes ergibt. Sofern das betreffende Material nicht unter der genannten Creative Commons Lizenz steht und die betreffende Handlung nicht nach gesetzlichen Vorschriften erlaubt ist, ist für die oben aufgeführten Weiterverwendungen des Materials die Einwilligung des jeweiligen Rechteinhabers einzuholen.

Weitere Details zur Lizenz entnehmen Sie bitte de Lizenzinformation auf http://creativecommons.org/ licenses/by/4.0/deed.de.

\section{Literatur}

1. BrayF,Ferlay J, Soerjomataram I, Siegel RL, TorreLA, Jemal A (2018) Global cancer statistics 2018: Globocan estimates of incidence and mortality worldwide for 36 cancers in 185 countries. CA Cancer J Clin 68(6):394-424. https://doi.org/10 3322/caac. 21492

2. Siegel RL, Miller KD, Jemal A (2019) Cancer statistics, 2019. CA Cancer J Clin 69(1):7-34 https://doi.org/10.3322/caac.21551

3. Kaatsch $P$, Spix $C$, Katalinic $A$, Hentschel $S$, Luttmann S, Waldeyer-Sauerland M, Waldmann A, Christ M, Folkerts J, Hansmann J, Gesellschaft der Epidemiologischen Krebsregister in Deutschland eV (2019) Krebs in Deutschland 2015/16. RobertKoch-Institut, Berlin, Germany

4. Statistik Austria (2020) Österreichisches Krebsregister (Stand 17.12.2020) und Todesursachenstatistik. http://statistik.at/web de/statistiken/menschen_und_gesellschaft/ gesundheit/krebserkrankungen/prostata/ 021791.html.Zugegriffen: 15. Febr. 2021

5. Schwenck J, Rempp H, Reischl G, Kruck S, Stenzl A, Nikolaou K, Pfannenberg C, la Fougère C (2017) Comparison of (68)Ga-labelled PSMA-11 and (11)C-choline in the detection of prostate cancer metastases by PET/CT. Eur J Nucl Med Mol Imaging 44(1):92-101. https://doi.org/10.1007/s00259016-3490-6

6. Silver DA, Pellicer I, Fair WR, Heston WD, CordonCardo C (1997) Prostate-specific membrane antigen expression in normal and malignant human tissues. Clin Cancer Res 3(1):81-85

7. Emmett L, Yin C, Crumbaker M, Hruby G, Kneebone A, Epstein R, Nguyen Q, Hickey A Ihsheish N, O'Neill G, Horvath L, Chalasani V, Stricker P, Joshua AM (2019) Rapid modulation of PSMA expression by androgen deprivation: serial (68)ga-PSMA-11 PET in men with hormonesensitive and castrate-resistant prostate cancer commencing androgen blockade. J Nucl Med 60(7):950-954. https://doi.org/10.2967/jnumed. 118.223099

8. Rowe SP, Gorin MA, Allaf ME, Pienta KJ, Tran PT, Pomper MG, Ross AE, Cho SY (2016) PET imaging of prostate-specific membrane antigen in prostate cancer: current state of the art and future challenges. Prostate Cancer Prostatic Dis 19(3):223-230. https://doi.org/10.1038/pcan. 2016.13

9. Prasad V, Steffen IG, Diederichs G, Makowski MR, Wust P, Brenner W (2016) Biodistribution of [(68)ga]PSMA-HBED-CC in patients with prostate cancer: characterization of uptake in normal organs and tumour lesions. Mol Imaging Biol 18(3):428-436. https://doi.org/10.1007/s11307016-0945-x
10. Afshar-Oromieh A, Malcher A, Eder M, Eisenhut $M$, LinhartHG, HadaschikBA, Holland-Letz T, GieselFL, Kratochwil C, Haufe S, Haberkorn U, Zechmann CM (2013) PET imaging with a [68Ga]gallium-labelled PSMA ligand for the diagnosis of prostate cancer: biodistribution in humans and first evaluation of tumour lesions. Eur J Nucl Med Mol Imaging 40(4):486-495. https://doi.org/10.1007/s00259012-2298-2

11. Fendler WP, Eiber $M$, Beheshti $M$, Bomanji J, Ceci F, Cho S, Giesel F, Haberkorn U, Hope TA, Kopka K, Krause BJ, Mottaghy FM, Schöder H, Sunderland J, WanS, Wester HJ, Fanti S, Herrmann K (2017) (68)Ga-PSMA PET/CT: joint EANM and SNMMI procedure guideline for prostate cancer imaging: version 1.0. Eur J Nucl Med Mol Imaging 44(6):1014-1024. https://doi.org/10. 1007/s00259-017-3670-z

12. Rischpler C, Beck TI, Okamoto S, Schlitter AM, Knorr K, Schwaiger M, Gschwend J, Maurer T, Meyer PT, Eiber M (2018) (68)Ga-PSMA-HBED-CC uptake in cervical, celiac, and sacral ganglia as an important pitfall in prostate cancer PET imaging. J Nucl Med 59(9):1406-1411. https://doi.org/10. 2967/jnumed.117.204677

13. de Galiza Barbosa F, Queiroz MA, Nunes RF, Costa LB, Zaniboni EC, Marin JFG, Cerri GG, Buchpiguel CA (2020) Nonprostatic diseases on PSMA PET imaging: a spectrum of benign and malignant findings. Cancer Imaging 20(1):23. https://doi.org/10.1186/s40644-020-00300-7

14. Kesch C, Kratochwil C, Mier W, Kopka K, Giesel FL (2017) (68)Ga or (18)F for prostate cancer imaging? J Nucl Med 58(5):687-688. https://doi.org/10. 2967/jnumed.117.190157

15. Giesel FL, Hadaschik B, Cardinale J, Radtke J, Vinsensia M, Lehnert W, Kesch C, Tolstov Y, Singer S, Grabe N, Duensing S, Schäfer M, Neels OC, Mier W, Haberkorn U, Kopka K, Kratochwil C (2017) F-18 labelled PSMA-1007: biodistribution, radiation dosimetry and histopathological validation of tumor lesions in prostate cancer patients. Eur J Nucl Med Mol Imaging 44(4):678-688. https://doi.org/ 10.1007/s00259-016-3573-4

16. Deutsche Krebsgesellschaft, AWMF (2019) Interdisziplinäre Leitlinie der Qualität S3 zur Früherkennung, Diagnose und Therapie der verschiedenen Stadien des Prostatakarzinoms (Langversion 5.1, AWMF Registernummer:043/022OL)

17. Eiber M, Weirich G, Holzapfel K, Souvatzoglou M, Haller B, Rauscher I, Beer AJ, Wester HJ, Gschwend J, Schwaiger M, Maurer T (2016) Simultaneous (68)Ga-PSMA HBED-CC PET/MRI improves the localization of primary prostate cancer. Eur Urol 70(5):829-836. https://doi.org/10.1016/j.eururo. 2015.12 .053

18. Giesel FL, Sterzing F, Schlemmer HP, HollandLetz T, Mier W, Rius M, Afshar-Oromieh A, Kopka K, Debus J, Haberkorn U, Kratochwil C (2016) Intra-individual comparison of (68)Ga-PSMA-11 $\mathrm{PET} / \mathrm{CT}$ and multi-parametric MR for imaging of primary prostate cancer. Eur J Nucl Med Mol Imaging 43(8):1400-1406. https://doi.org/10. 1007/s00259-016-3346-0

19. Maurer T, Gschwend JE, Rauscher I, Souvatzoglou M, Haller B, Weirich G, Wester HJ, Heck M, Kübler H, Beer AJ, Schwaiger M, Eiber M (2016) Diagnostic efficacy of (68)gallium-PSMA positron emission tomography compared to conventional imaging forlymph nodestaging of 130 consecutive patients with intermediate to high risk prostate cancer. J Urol 195(5):1436-1443. https://doi.org/ 10.1016/j.juro.2015.12.025
20. Pyka T, Okamoto $S$, Dahlbender $M$, Tauber $R$ Retz M, Heck M, Tamaki N, Schwaiger M, Maurer T, Eiber M (2016) Comparison of bone scintigraphy and (68)Ga-PSMA PET for skeletal staging in prostate cancer. Eur J Nucl Med Mol Imaging 43(12):2114-2121. https://doi.org/10. 1007/s00259-016-3435-0

21. Hofman MS, Lawrentschuk N, Francis RJ, Tang C Vela I, Thomas P, Rutherford N, Martin JM, Frydenberg M, Shakher R, et al. (2020) Prostatespecific membrane antigen PET-CT in patients with high-risk prostate cancer before curativeintent surgery or radiotherapy (proPSMA): a prospective, randomised, multicentre study. Lancet 395(10231):1208-1216. https://doi.org/10.1016/ s0140-6736(20)30314-7

22. Cornford $P$, van den Bergh RC, Briers $E$, Van den Broeck T, Cumberbatch MG, De Santis M, Fanti S, Fossati N, Gandaglia G, Gillessen S (2021) EAUEANM-ESTRO-ESUR-SIOG guidelines on prostate cancer. Part II-2020 update: treatment of relapsing and metastatic prostate cancer. Eur Urol 79(2):263-282. https://doi.org/10.1016/j.eururo. 2020.09.046

23. Farolfi A, Gafita A, Calais J, Eiber M, AfsharOromieh A, Spohn F, Barbato F, Weber M, Ilhan H, Cervati V, Wetter A, Hadaschik B, Briganti A, Walz J, Pianori D, Fanti $S$, Haberkorn U, Herrmann K, Fendler WP (2019) (68)Ga-PSMA-11 positron emission tomography detects residual prostate cancer after prostatectomy in a multicenter retrospective study. J Urol 202(6):1174-1181. https://doi.org/10.1097/ju.0000000000000417

24. Perera M, Papa N, Christidis D, Wetherell D, Hofman MS, Murphy DG, Bolton D, Lawrentschuk N (2016) Sensitivity, specificity, and predictors of positive (68)Ga-prostate-specific membrane antigen positron emission tomography in advanced prostate cancer: a systematic review and metaanalysis. Eur Urol 70(6):926-937. https://doi.org/ 10.1016/j.eururo.2016.06.021

25. Schmidt-Hegemann NS, Fendler WP, Buchner A Stief C, Rogowski P, Niyazi M, Eze C, Li M, Bartenstein P, Belka C, Ganswindt U (2017) Detection level and pattern of positive lesions using PSMA PET/CT for staging prior to radiation therapy. Radiat Oncol 12(1):176. https://doi.org/ 10.1186/s13014-017-0902-0

26. Mottet N, van den Bergh RC, Briers E, Van den Broeck T, Cumberbatch MG, De Santis M, Fanti S Fossati N, Gandaglia G, Gillessen S (2021) EAUEANM-ESTRO-ESUR-SIOG guidelines on prostate cancer-2020 update. Part 1: screening, diagnosis, and local treatment with curative intent. Eur Urol 79(2):243-262. https://doi.org/10.1016/j.eururo. 2020.09.042

27. Roach PJ, Francis R, Emmett L, Hsiao E, Kneebone A, Hruby G, Eade T, Nguyen QA, Thompson BD, Cusick T, McCarthy M, Tang C, Ho B, Stricker PD Scott AM (2018) The impact of (68)Ga-PSMA $\mathrm{PET} / \mathrm{CT}$ on management intent in prostate cancer: results of an Australian prospective multicenter study. J Nucl Med 59(1):82-88. https://doi.org/10. 2967/jnumed.117.197160

28. Giesel FL, Fiedler $H$, Stefanova $M$, Sterzing $F$, Rius M, Kopka K, Moltz JH, Afshar-Oromieh A, Choyke PL, Haberkorn U, Kratochwil C (2015) PSMA PET/CT with Glu-urea-Lys-(Ahx)-[(68)Ga(HBEDCC)] versus $3 D C T$ volumetric lymph node assessment in recurrent prostate cancer. Eur J Nucl Med Mol Imaging 42(12):1794-1800. https://doi. org/10.1007/s00259-015-3106-6

29. Reyes DK, Pienta KJ (2015) The biology and treatment of oligometastatic cancer. Oncotar- 
get 6(11):8491-8524. https://doi.org/10.18632/ oncotarget.3455

30. Afshar-Oromieh A, Avtzi $E$, Giesel FL, HollandLetz T, Linhart HG, Eder M, Eisenhut M, Boxler S, Hadaschik BA, Kratochwil C, Weichert W, Kopka K, Debus J, Haberkorn U (2015) The diagnostic value of PET/CT imaging with the (68)Ga-labelled PSMA ligand HBED-CC in the diagnosis of recurrent prostate cancer. Eur J Nucl Med Mol Imaging 42(2):197-209. https://doi.org/10.1007/s00259014-2949-6

31. Hijazi S, Meller B, Leitsmann C, Strauss A, Meller J, Ritter CO, Lotz J, Schildhaus HU, Trojan L, Sahlmann CO (2015) Pelvic lymph node dissection for nodal oligometastatic prostate cancer detected by 68Ga-PSMA-positron emission tomography/computerized tomography. Prostate 75(16):1934-1940. https://doi.org/10.1002/pros. 23091

32. Deutsche Gesellschaft für Nuklearmedizin eV (2016) Therapie mit Lu-177-PSMA, Dosimetrie und Nachsorge beim metastasierten kastrationsresistenten Prostatakarzinom - Konsensusempfehlung https://doi.org/10.1007/s00259-008-0778-1

33. Habl G, Sauter K, Schiller K, Dewes S, Maurer T, Eiber M, Combs SE (2017) (68) Ga-PSMA-PET for radiation treatment planning in prostate cancer recurrences after surgery: individualized medicine or new standard in salvage treatmen Prostate 77(8):920-927. https://doi.org/10.1002/ pros. 23347

34. van Leeuwen PJ, Stricker $P$, Hruby G, Kneebone $A$, Ting $F$, Thompson B, Nguyen $Q$, Ho B, Emmett $L$ (2016) (68) Ga-PSMA has a high detection rate of prostate cancer recurrence outside the prostatic fossa in patients being considered for salvage radiation treatment. BJU Int 117(5):732-739. https://doi.org/10.1111/bju.13397

35. Afshar-Oromieh A, Holland-Letz T, Giesel FL, Kratochwil C, Mier W, Haufe S, Debus N, Eder M, Eisenhut M, Schäfer M, Neels $O$, Hohenfellner $M$, Kopka K, Kauczor HU, Debus J, Haberkorn U (2017) Diagnostic performance of (68)Ga-PSMA11 (HBED-CC) PET/CT in patients with recurrent prostate cancer: evaluation in 1007 patients. Eur J Nucl Med Mol Imaging 44(8):1258-1268. https:// doi.org/10.1007/s00259-017-3711-7

36. Eiber M, Herrmann K, Calais J, Hadaschik B, Giesel FL, Hartenbach M, Hope T, Reiter R, Maurer T, Weber WA, Fendler WP (2018) Prostate cancer molecular imaging standardized evaluation (PROMISE): proposed miTNM classification for the interpretation of PSMA-ligand PET/CT. J Nucl Med 59(3):469-478. https://doi.org/10.2967/jnumed. 117.198119

37. Weinreb JC, Barentsz JO, Choyke PL, Cornud F, Haider MA, Macura KJ, Margolis D, Schnall MD, Shtern F, Tempany CM, Thoeny HC, Verma S (2016) PI-RADS prostate imaging - reporting and data system: 2015, version 2. Eur Urol 69(1):16-40. https://doi.org/10.1016/j.eururo.2015.08.052

38. Rowe SP, Pienta KJ, Pomper MG, Gorin MA (2018) PSMA-RADS version 1.0: a step towards standardizing the interpretation and reporting of PSMA-targeted PET imaging studies. Eur Uro 73(4):485-487. https://doi.org/10.1016/j.eururo. 2017.10.027

39. Grubmüller B, Baltzer P, Hartenbach S, D'Andrea D, Helbich TH, Haug AR, Goldner GM, Wadsak W Pfaff S, Mitterhauser M, et al. (2018) PSMA ligand PET/MRI for primary prostate cancer: staging performance and clinical impact. Clin Cancer Res 24(24):6300-6307.https://doi.org/10.1158/10780432. Ccr-18-0768
40. Kratochwil C, Fendler WP, Eiber M, Baum R, Bozkurt MF, Czernin J, Delgado Bolton RC, Ezziddin S, Forrer F, et al. (2019) EANM procedure guidelines for radionuclide therapy with (177)Lulabelled PSMA-ligands ((177)Lu-PSMA-RLT). Eur J Nucl Med Mol Imaging 46(12):2536-2544. https:// doi.org/10.1007/s00259-019-04485-3

41. van Kalmthout LWM, Lam M, deKeizer B, Krijger GC, Ververs TFT, de Roos R, Braat A (2018) Impact of external cooling with icepacks on (68)Ga-PSMA uptake in salivary glands. EJNMMI Res 8(1):56. https://doi.org/10.1186/s13550-018-0408-2

42. Delker A, Fendler WP, Kratochwil C, Brunegraf $A$, Gosewisch A, Gildehaus FJ, Tritschler S, Stief CG Kopka K, Haberkorn U, Bartenstein P, Böning G (2016) Dosimetry for (177)Lu-DKFZ-PSMA-617: a new radiopharmaceutical for the treatment of metastatic prostate cancer. Eur J Nucl Med Mol Imaging 43(1):42-51. https://doi.org/10.1007/ s00259-015-3174-7

43. Yordanova A, Becker A, EppardE, Kürpig S, Fisang C, Feldmann G, Essler M, Ahmadzadehfar H (2017) The impact of repeated cycles of radioligand therapy using [(177)Lu]Lu-PSMA-617 on renal function in patients with hormone refractory metastatic prostate cancer. Eur J Nucl Med Mo Imaging 44(9):1473-1479. https://doi.org/10. 1007/s00259-017-3681-9

44. Bräuer A, Grubert LS, Roll W, Schrader AJ, Schäfers M, Bögemann M, Rahbar K (2017) 177LuPSMA-617 radioligand therapy and outcome in patients with metastasized castration-resistant prostate cancer. Eur J Nucl Med Mol Imaging 44(10):1663-1670. https://doi.org/10.1007/ s00259-017-3751-z

45. Ahmadzadehfar $\mathrm{H}$, Wegen $\mathrm{S}$, Yordanova $A$ Fimmers R, KürpigS, EppardE, WeiX, SchlenkhoffC, Hauser S, Essler M (2017) Overall survival and response pattern of castration-resistant metastatic prostate cancer to multiple cycles of radioligand therapy using 177LuLu-PSMA-617. Eur J Nucl Med Mol Imaging 44(9):1448-1454. https://doi.org/10. 1007/s00259-017-3716-2

46. Rathke H, Giesel FL, Flechsig P, Kopka K, Mier W, Hohenfellner M, Haberkorn U, Kratochwil C (2018) Repeated (177)Lu-labeled PSMA-617 radioligand therapy using treatment activities of up to $9.3 \mathrm{GBq}$ J Nucl Med 59(3):459-465. https://doi.org/10. 2967/jnumed.117.194209

47. Rahbar K, Ahmadzadehfar $\mathrm{H}$, Kratochwil $\mathrm{C}$, Haberkorn U, Schäfers M, Essler M, Baum RP Kulkarni HR, Schmidt M, Drzezga A, et al. (2017) German multicenter study investigating 177LuPSMA-617 radioligand therapy in advanced prostate cancer patients. J Nucl Med 58(1):85-90. https://doi.org/10.2967/jnumed.116.183194

48. Hofman MS, Violet J, Hicks RJ, Ferdinandus J, Thang SP, Akhurst T, Iravani A, Kong G, Ravi Kumar A, Murphy DG, Eu P, Jackson P, Scalzo M Williams SG, Sandhu S (2018) [(177)Lu]-PSMA-617 radionuclide treatment in patients with metastatic castration-resistant prostate cancer (LuPSMA trial): a single-centre, single-arm, phase 2 study. Lancet Oncol 19(6):825-833. https://doi.org/10.1016/ s1470-2045(18)30198-0

49. Heck MM, Retz M, D'Alessandria C, Rauscher I, Scheidhauer K, Maurer T, Storz E, Janssen F, Schottelius M, Wester HJ, Gschwend JE, Schwaiger M, Tauber R, Eiber M (2016) Systemic radioligand therapy with (177)Lu labeled prostate specific membrane antigen ligand for imaging and therapy in patients with metastatic castration resistant prostate cancer.JUrol 196(2):382-391. https://doi. org/10.1016/j.juro.2016.02.2969
50. Baum RP, Kulkarni HR, Schuchardt $C$, Singh A, Wirtz M, Wiessalla S, Schottelius M, Mueller D, Klette I, Wester HJ (2016) 177Lu-labeled prostatespecific membrane antigen radioligand therapy of metastatic castration-resistant prostate cancer: safety and efficacy. J Nucl Med 57(7):1006-1013. https://doi.org/10.2967/jnumed.115.168443

51. Kratochwil C, Giesel FL, Stefanova M, Benesova M, Bronzel M, Afshar-Oromieh A, Mier W, Eder M, Kopka K, Haberkorn U (2016) PSMA-targeted radionuclide therapy of metastatic castrationresistant prostate cancer with $177 \mathrm{Lu}$-labeled PSMA-617. J Nucl Med 57(8):1170-1176. https:// doi.org/10.2967/jnumed.115.171397

52. Ahmadzadehfar $H$, Rahbar K, Kürpig $S$, Bögemann M, Claesener M, Eppard E, Gärtner F, Rogenhofer S, Schäfers M, Essler M (2015) Early side effects and first results of radioligand therapy with (177)Lu-DKFZ-617 PSMA of castrate-resistant metastatic prostate cancer: a two-centre study. EJNMMI Res 5(1):114. https://doi.org/10.1186/ s13550-015-0114-2

53. von Eyben FE, Roviello G, Kiljunen T, Uprimny $C$, Virgolini I, Kairemo K, Joensuu T (2018) Third-line treatment and (177)Lu-PSMA radioligand therapy of metastatic castration-resistant prostate cancer: a systematic review. Eur J Nucl Med Mol Imaging 45(3):496-508. https://doi.org/10.1007/s00259017-3895-x

54. Rahbar K, Bode A, Weckesser M, Avramovic N, Claesener M, Stegger L, Bögemann M (2016) Radioligand therapy with 177Lu-PSMA-617 as a novel therapeutic option in patients with metastatic castration resistant prostate cancer Clin Nucl Med 41(7):522-528. https://doi.org/10 1097/rlu.0000000000001240

55. Rahbar K, Schmidt M, Heinzel A, Eppard E, Bode A, Yordanova A, Claesener M, Ahmadzadehfar $\mathrm{H}$ (2016) Response and tolerability of a single dose of 177Lu-PSMA-617 in patients with metastatic castration-resistant prostate cancer: a multicenter retrospective analysis. J Nucl Med 57(9):1334-1338. https://doi.org/10.2967/jnumed.116.173757

56. Yadav MP, Ballal S, Tripathi M, Damle NA, Sahoo RK, Seth A, Bal C (2017) (177)Lu-DKFZPSMA-617 therapy in metastatic castration resistant prostate cancer: safety, efficacy, and quality of life assessment. Eur J Nucl Med Mol Imaging 44(1):81-91. https://doi.org/10.1007/ s00259-016-3481-7

57. Fendler WP, Reinhardt $S$, Ilhan $H$, Delker A, Böning G, Gildehaus FJ, Stief C, Bartenstein P, Gratzke C, Lehner S, Rominger A (2017) Preliminary experience with dosimetry, response and patient reported outcome after 177Lu-PSMA-617 therapy for metastatic castration-resistant prostate cancer. Oncotarget 8(2):3581-3590. https://doi.org/10. 18632/oncotarget.12240

58. Kratochwil C, Bruchertseifer $F$, Rathke $H$, Hohenfellner M, Giesel FL, Haberkorn U, Morgenstern A (2018) Targeted a-therapy of metastatic castration-resistant prostate cancer with 225Ac-PSMA617: swimmer-plot analysis suggests efficacy regarding duration of tumor control. J Nucl Med 59(5):795-802

59. SathekgeM,BruchertseiferF,Knoesen O, ReynekeF, Lawal I, Lengana T, Davis C, Mahapane J, Corbett C, Vorster M, Morgenstern A (2019) 225Ac-PSMA-617 in chemotherapy-naive patients with advanced prostate cancer: a pilot study. Eur J Nucl Med Mol Imaging 46(1):129-138. https://doi.org/10.1007/ s00259-018-4167-0

60. Sathekge M, Bruchertseifer F, Vorster M, Lawal IO, Knoesen O, Mahapane J, Davis C, Reyneke F, 
Maes A, Kratochwil C, Lengana T, Giesel FL, Van de Wiele C, Morgenstern A (2020) Predictors of overall and disease-free survival in metastatic castration-resistant prostate cancer patients receiving 225ac-PSMA-617 radioligand therapy. J Nucl Med 61(1):62-69. https://doi.org/10.2967/ jnumed.119.229229

61. Ahmadzadehfar $H$, Rahbar K, Baum RP, Seifert R, Kessel K, Bögemann M, Kulkarni HR, Zhang J, Gerke C, Fimmers R (2020) Prior therapies as prognostic factors of overall survival in metastatic castration-resistant prostate cancer patients treated with [177 Lu] Lu-PSMA-617. A WARMTH multicenter study (the 617 trial). Eur J Nucl Med Mol Imaging. https://doi.org/10.1007/s00259020-04797-9

Hinweis des Verlags. Der Verlag bleibt in Hinblick auf geografische Zuordnungen und Gebietsbezeichnungen in veröffentlichten Karten und Institutsadressen neutral.

\section{Neuerscheinungen in der Urologie}

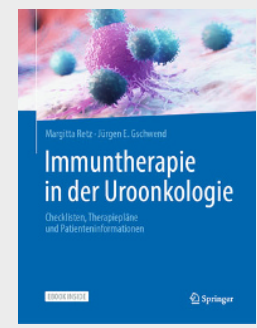

\section{Immuntherapie in der Uroonkologie}

Retz, M., Gschwend, J. E.

1.Auflage, 365 Seiten, inkl. Ebook, 79,99EUR, ISBN 978-3-662-60977-4

Vereinfacht dem Arzt die onkologische Therapie durch klare Handlungsanweisungen in Form von Checklisten, Patienteninformationsblätter und Therapieplänen

In einzigartiger Form bündelt das Buch das aktuelle Wissen, welches der Arzt für die sichere Behandlung zur Versorgung von urologischen Tumorpatienten benötigt. Zahlreiche Checklisten,

Tabellen, Therapiepläne und Patienteninformationen machen ein schnelles Nachschlagen möglich. Erstmalig wurde das Nebenwirkungsmanagement von immunvermittelten Toxizitäten auf der Grundlage von internationalen Leitlinien in Tabellenform aufgelistet. Weitere Schwerpunkte sind die Pharmakologie von Immun- und Targettherapeutika unter besonderer Berücksichtigung der Nebenwirkungsprofile und Kontraindikationen.

Die übersichtlich gestalteten Therapieschemata und Patienteninformationen können als Vorlage für die eigene Arbeit genutzt werden - sie sind druckbar und kopierbar. Für den direkten Einsatz während der Therapie erhalten Sie zusätzlich das eBook.

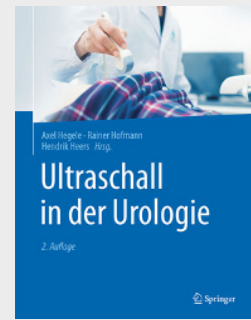

\section{Ultraschall in der Urologie}

Hegele, A., Hofmann, R., Heers, $H$.

2.Auf., 339 Seiten, 129,99EUR (April 2021) ISBN 978-3-662-60396-3

Der vorliegende Atlas ist Lehrbuch und Nachschlagewerk zugleich. Er liefert dem klinisch tätigen Urologen einen reichen Fundus von über 400 Referenzaufnahmen häufiger Befunde und interessanter Raritäten. Neben technischen Grundlagen der Sonographie sowie praktischen Tipps zur Vermeidung von Fehlbefunden finden Sie umfangreiche Informationen zur sonographischen Diagnostik benigner und maligner urologischer Erkrankungen. Auf Normbefunde und Normvarianten wird zum besseren Verständnis gezielt eingegangen. Aktuelle Forschungsergebnisse und differenzialdiagnostische Tabellen zur schnellen Kontrolle der gestellten Diagnose runden das Werk ab. Die Auswahl der Bilder folgte höchsten Qualitätsansprüchen. Komplexe Befunde werden zusätzlich durch aussagekräftige Graphiken veranschaulicht.

„Ultraschall in der Urologie", ein unverzichtbarer Begleiter für alle, die sich mit der sonographischen Diagnostik des Urogenitaltrakts befassen.

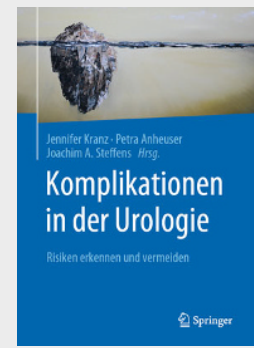

\section{Komplikationen in der Urologie}

Kranz, J., Anheuser, P., Steffens, J. A.

1.Aufl., 478 Seiten, 79,99 EUR (April 2021) ISBN 978-3-662-60624-7 Systematische Darstellung von Komplikationen in der diagnostischen, konservativen und operativen Urologie

Dieses Buch schildert Komplikationen und Zwischenfälle aus dem gesamten Fachbereich der Urologie. Neben konservativen und operativen Therapieoptionen werden auch die Indikationsstellung verschiedener Verfahren sowie diagnostische Maßnahmen einbezogen. Unter Berücksichtigung des aktuellen Wissenstandes bietet das Buch Strategien zur Vermeidung und Therapievorschläge zur Beherrschung von Komplikationen. Häufigkeitsangaben typischer, aber auch seltener Komplikationen werden systematisch aufgeführt. Der urologisch tätige Arzt erhält Tipps für ein strukturiertes Risikomanagement.

Für alle operativ tätigen Urologen und niedergelassenen Fachärzte, aber auch zur Erstellung von Gutachten, dient es als wertvolle Lektüre. 